\title{
Provenance and tectonic settings of accretionary wedge sediments on northeastern Karaginski Island (Kamchatka, Russian Far East)
}

\author{
G. V. Ledneva ${ }^{1}$, J. I. Garver ${ }^{2}$, M. N. Shapiro ${ }^{3}$, J. Lederer ${ }^{2}$, M. T. Brandon ${ }^{4}$, and \\ K. T. Hollocher ${ }^{2}$
}

\begin{abstract}
The provenance and tectonic setting of the sediments and rare cobbles from the accretionary wedge on Karaginski Island were examined. This study provides a picture of the nature of the NE Asian paleomargin in the middle Eocene to early Miocene. The trace element geochemistry of shale from the flysch and mélange indicates (a) their common provenance with the quartzofeldspathic and arkosic sandstones, and (b) derivation of these sediment types from active continental and island arcs with partially dissected basement rocks. Previous studies of the sandstones [Shapiro et al., 2000] showed that they dominate in debris whose composition is very similar to those of the felsic extrusive and intrusive rocks in cobbles and boulders embedded in the sediments. Fission-track (FT) ages from detrital zircon from sandstones, and felsic cobbles show their derivation from sources with several cooling histories in the Late Cretaceous and Early Tertiary. The youngest detrital component probably represents continuous younging population from active volcanism in the source that is nearly the same age of deposition. The felsic cobbles represent the essentially random selection of the older material component. They include three distinct types: (a) subduction-related low-K tonalites and trondhjemites and their extrusive analogues; (b) crustally-derived med-K two-mica leucogranites, and (c) crustally-derived high-K biotite leucogranites. The felsic rock cobbles of all types have a narrow range of zircon FT grain ages that indicate cooling in the source in the Late Cretaceous to Early Paleogene. Tonalites and trondhjemites (low-K) cooled at $53.8(-3.5 /+3.8)$ Ma and 78.5 $(-6.7 /+7.6)$ Ma. The rhyolites $($ med-K) cooled at $70.1(-3.7 /+3.9)$ Ma. The biotite leucogranites (high-K) cooled between 59.1 Ma $(-3.3 /+3.9)$ and $58.6(-3.2 /+3.3)$ Ma (two clasts). The Late Cretaceous cooling ages (70-78 Ma) probably reflect original magmatic activity whereas the Early Eocene cooling ages may indicate timing of exhumation. The chemical and age data obtained on the felsic cobbles suggest derivation of the accretionary wedge sediments from a source formed either by: (a) subduction in the Late Cretaceous and collision/exhumation in the Early Paleogene; or by (b) continuous subduction. The closest petrochemical and age intrusive and extrusive analogues of the felsic cobbles occur only locally in the Olyutorsky Peninsula subterrane. Nonetheless, this structure is too small and too remote from Karaginski Island to supply enough material for the middle Eocene-early Miocene accretionary wedge sediments. We speculate that the structure of the Olyutorsky Peninsula subterrane might be extended in the shelf where several highs exist.
\end{abstract}

\footnotetext{
${ }^{1}$ Institute of the Lithosphere of Marginal Seas, Moscow, Russia

2 Geology Department, Union College, Schenectady, USA

3 Institute of the Earth Physics after A. A. Gamburtsev, Moscow, Russia

4 Dept. of Geology and Geophysics, Yale University, New Haven, USA
}

Copyright 2004 by the Russian Journal of Earth Sciences.

Paper number TJE04149.

ISSN: $1681-1208$ (online)

The online version of this paper was published 3 June 2004. URL: http://rjes.wdcb.ru/v06/tje04152/tje04152.htm

\section{Introduction}

One of the vexing problems with sediments deposited and imbricated along an active margin is that source rocks can consist of a complicated assemblage of volcano-plutonic material that is more or less active at the time of deposition. However detritus from ancient volcano-plutonic sequences can also form a significant percentage of the sediment. Mixed together, volcanic-plutonic detritus may record a full and complicated history of igneous activity along the margin. Through a combination of geochemistry, petrological modeling, and age dating, one can establish constraints on possible 


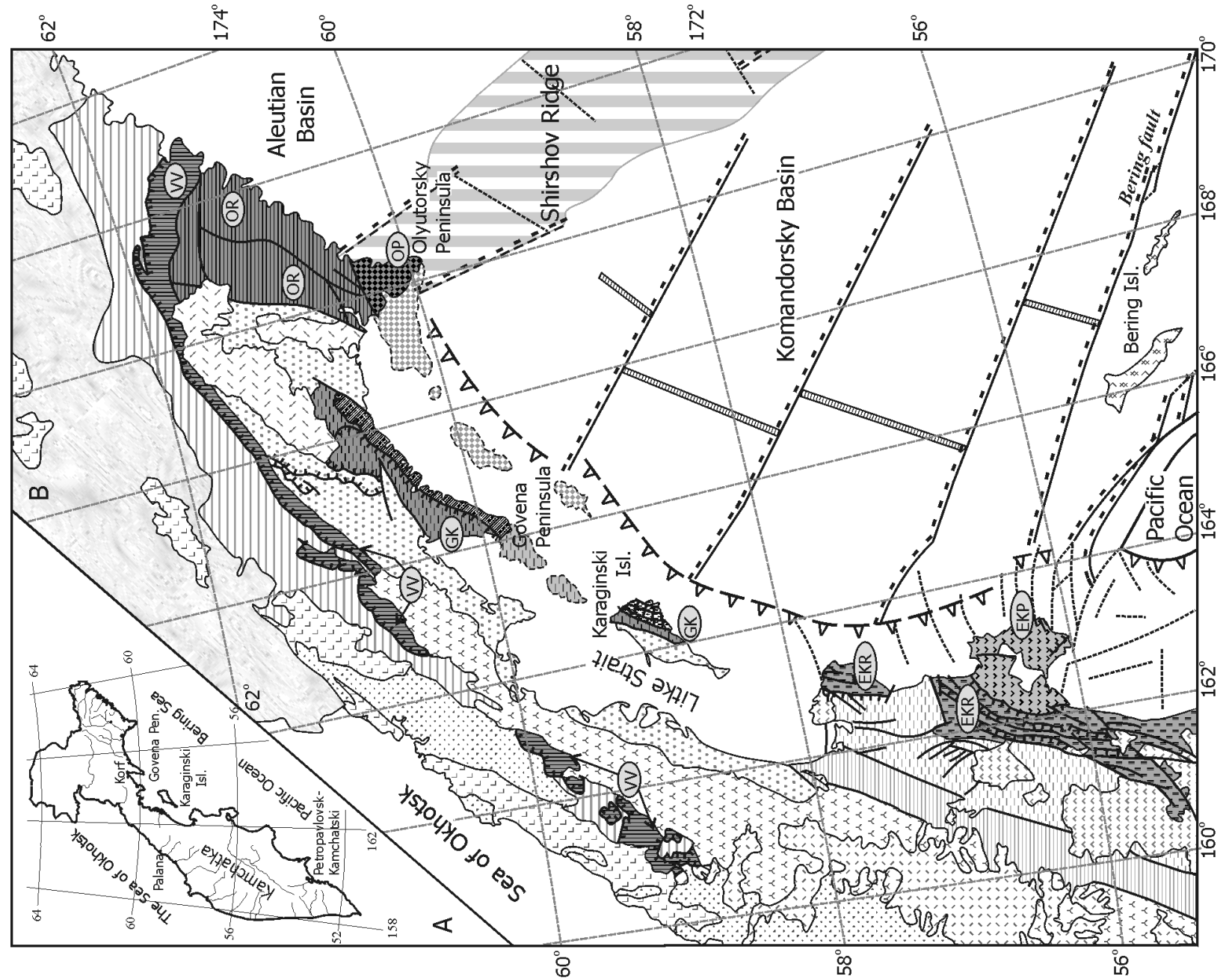


source terrains and how the margin developed though time. Along a margin like Kamchatka, such an analysis is crucial because there has been nearly $100 \mathrm{Ma}$ of continuous volcanism that has been punctuated by the collision of far-traveled island-arc terranes.

In this paper, we try (a) to demonstrate that the shale and sandstone composing the matrix of the accretionary wedge units on Karaginski Island have the common source, and (b) to reconstruct both ancient and active at the time provenance for these sediments. For a long time it was recognized that the studied sedimentary deposits contain cobbles of felsic extrusive and intrusive rocks; and sandstones are also abundant in detritus of petrographically similar igneous felsic rocks [Shapiro et al., 2000]. Our recent studies [Garver et al., 2001; Shapiro et al., 2004] proved that these sandstones and shales were deposited along the eastern Kamchatka continental margin through the middle Eocene to early Miocene time [Chekovich et al., 1990] while the cobbles incorporated in these sediments are the Late Cretaceous in age. Moreover, ages of the latter coincide with those of one of the older detrital components in the sandstones. This means that the information on the source of the older clastic material can be obtained through the direct studies of the felsic cobble geochemistry and the comparative analysis of the latter with rocks in possible sources. The youngest detrital component cannot be reconstructed though the direct examination of any material either composing or embedded into these sediments.

The provenance of the felsic igneous cobbles itself was a subject of long lasted dispute as the large volcanic - plutonic assemblage dominated in felsic igneous rocks was not recognized in the close vicinity to the Island. In the presentday geological structure, the ancient Late Cretaceous complexes that are exposed next to the accretionary wedge in Karaginski, are mainly composed of mafic to intermediate volcanics and ophiolites originally generated in suprasubduction and oceanic settings and represent the fragments of far-traveled terranes [Chamov, 1996; Chekhovich et al., 1990; Kravchenko-Berezhnoy and Nazimova, 1991], probably, accreted to paleo-Asian continental margin in the Eocene time [Soloviev et al., 1998, 2002]. The following suggestions were made on a provenance of the cobbles and accretionary wedge sediments [Shapiro et al., 2000]: (a) they represent the material transported on the oceanic plate from the Shirshov Ridge; or (b) fragments of subduction-related continental margin volcanic-plutonic complex whose fragments are only locally preserved in the Olyutorsky Peninsula (Figure 1). This problem remains unresolved mainly because of lack of reliable data on geochemistry of both felsic cobbles and rocks exposed on the mainland and covered by waters of the Komandorsky basin.

In this provenance study we investigate the sources of sediments composing the transitional volcanic-sedimentary, flysch and mélange. We use the geochemistry of shales, geochemistry of felsic extrusive and intrusive rock cobbles. We combine this information with zircon fission-track ages of granitic and rhyolitic clasts as well as detrtial zircons from the sandstones. In doing so, we demonstrate that the shales, sandstone, and conglomerates has a similar provenance, and we reconstruct the paleogeology of the margin.
This reconstruction allows us to infer that a history that includes unique information on evolution of the provenance area whose relics are only locally preserved and whose geological history is poorly understood.

\section{Geological Framework and Tectonic Setting}

\section{General Tectonic Setting}

The general geological-tectonic map of the northern Kamchatka and Koryak Highlands is shown in Figure 1. From the middle to Late Cretaceous, a well-established Andean-style arc, referred to as the Okhotsk-Chukchi arc, existed to the west of Kamchatka on the NE Asian mainland. Simultaneously, the intraoceanic Olyutorsky arc superterrane (divided into the Vatyna-Vyvenka, Govena-Karaginski, Olyutorsky Ridge and Peninsula subterranes ${ }^{1}$ [Bogdanov and Khain, 2000b] formed well to the south. In the middle Eocene, the intraoceanic Olyutorsky arc superterrane collided with the Asian mainland [Soloviev et al., 1998, 2002], effectively adding this new Cretaceous-Paleocene volcanic arc to the margin. After collision, a new continental arc, referred to as the Western Kamchatka - Koryak volcanic belt, was established on top of this accreted superterrane (Olyutorsky), tectonically underlying it continental slope flysch deposits and outboard (east) of the Okhotsk-Chukchi arc. Detailed studies of chronology of Cenozoic volcanism on this belt indicate that volcanism was active in the Eocene (46.5 $\pm 0.3-37.4 \pm 0.6 \mathrm{Ma})$ [Bagdasaryan et al., 1994] and Oligocene [Achmetiev et al., 1989; Filatova, 1988] in its different segments. The termination of the volcanic activity in this belt in the early Oligocene was followed by establishing a new continental arc, referred to as the Sredinny Range belt, whose volcanics in the Kamchatka Isthmus and north of it are dated at the Miocene and Pliocene ( $\sim 15$ and $6 \mathrm{Ma}$ ) [Hochstaedter et al., 1994]. This accretionary complex on Karaginski Island is the leading edge of this continental margin that has been active since the Eocene.

The Miocene-Pliocene Sredinny Range volcanic belt may have been resulted from the westward subduction of the Komandorsky oceanic lithosphere beneath the Kamchatka. This is consistent with the initiation of spreading in the Komandorsky basin at 30-25 Ma [Baranov et al., 1991] and generation of the new oceanic crust in the Neogene [Bogdanov, 1988] as MORB-like basalts from DSDP site 191 were dated at $9.3 \pm 0.8 \mathrm{Ma}$ by K/Ar method [Rubenstone, 1984]. Gravity and seismic reflection data indicate that the continental margin to the east of Karaginski Island is underlain by an accretionary wedge and a buried trench at the base of the continental slope. The sediments filling this trench are the Middle Miocene and Pliocene-Pleistocene in age [Shipilov, 1988].

\footnotetext{
${ }^{1}$ Note that the Olyutorsky Ridge and Olyutorsky Peninsula subterranes are distinct from the "Olyutorsky superterrane" in the literature Provenance of Sediments of Northeastern Karaginski Island
} 


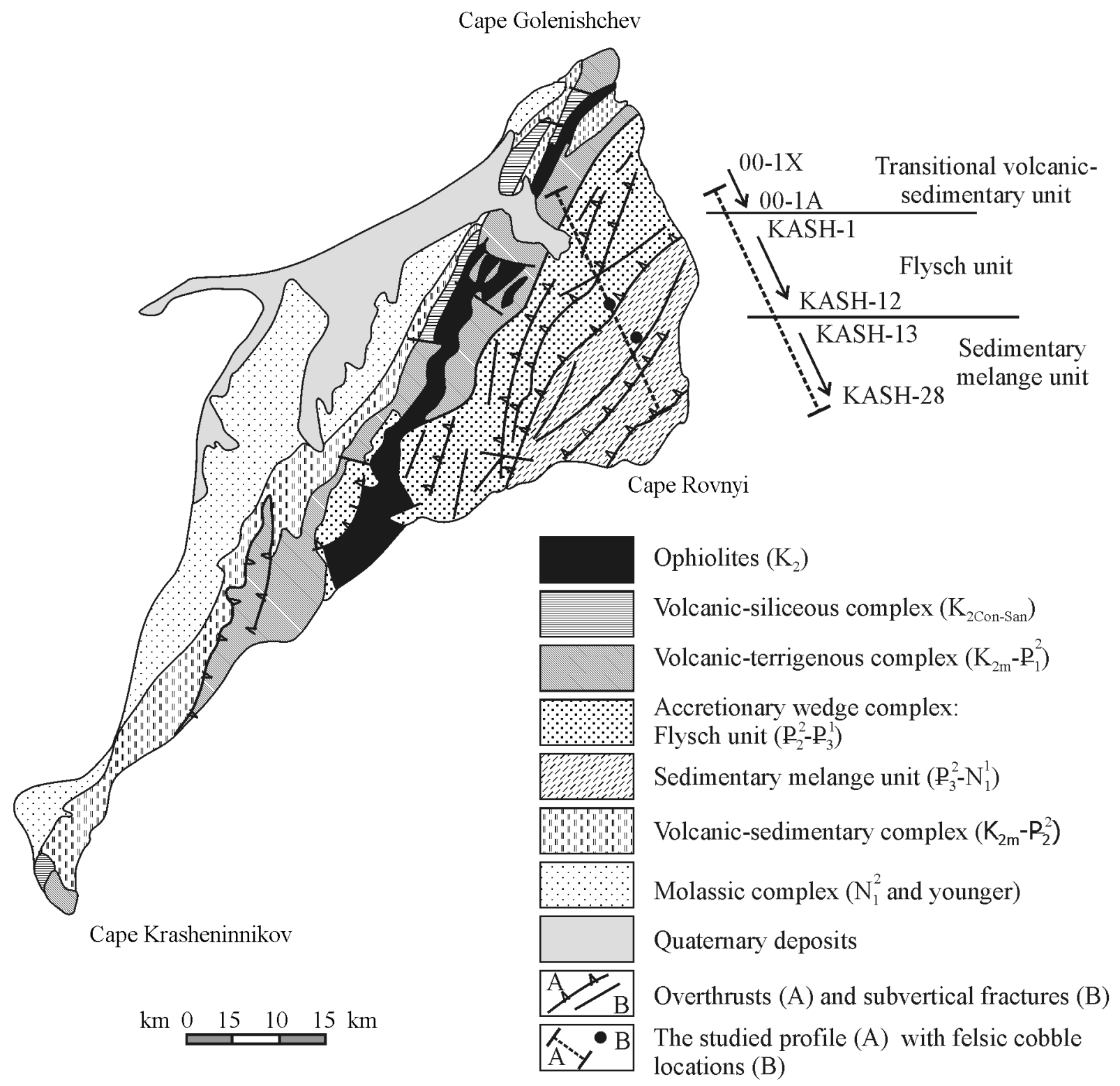

Figure 2. The geologic map of Karaginski Island (after [Chekhovich et al., 1990]) with sampling location sites. The location of Karaginski Island is shown in the Figure 1.

At the same time, the oldest sedimentary deposits found in the accretionary complex on Karaginski are dated at $46 \mathrm{Ma}$ [Garver et al., 2001; Shapiro et al., 2004]. The EarlyMiddle Eocene evolution of the Komandorsky segment separated in the present-day structure from the Pacific ocean by the Aleutian arc and from the Aleutian basin by the Shirshov Ridge is poorly understood. Nonetheless, it was suggested that the relic of the Kula plate existed to the west of the Shirshov Ridge and was subducted beneath the Kamchatka at this time [Baranov et al., 1991; Bogdanov and Khain, 2000b; Seliverstov, 1998]. Therefore, in this scenario, Karaginski Island represents a forearc high formed by west- ward subduction of the old and cold Kula oceanic plate in the Middle Eocene and the Komandorsky young and hot oceanic plate in the Middle Miocene-Pleistocene beneath the Kamchatka.

\section{General Geological Structure of the Island}

Karaginski Island is located in the western part of the Komandorsky basin and is separated from the mainland by the Litke Strait (Figure 1). The general geological structure 
of the island is shown in Figure 2. The west side of the Island is composed of Oligocene, Miocene and Pliocene volcaniclastic deposits of the Litke Basin which continues westward under the modern Litke Straight to the mainland [Chekhovich et al., 1990]. These deposits rest unconformably on relics of the larger Govena-Karaginski arc subterrane represented there by upper Cretaceous backarc volcanic-terrigenous sequences, upper Cretaceous and lower Paleogene island-arc volcanic-siliceous, volcanic-sedimentary and ophiolitic complexes [Chamov, 1996; Chekhovich et al., 1990; KravchenkoBerezhnoy and Nazimova, 1991]. The east side of the Island, whose deposits were the main objective of the present study, is dominated by middle Eocene to Oligocene terrigenous rocks divided into flysch and sedimentary mélange (hereinafter, "mélange") units [Chekhovich et al., 1990; Shapiro and Petrina, 1985]. The geological structure of the east side of Karaginski Island is complicated but similar to many accretionary wedges with imbricated slices of highly deformed sedimentary rocks and local mélange units all in what are generally seaward-dipping structural panels [Chekhovich et al., 1990].

The flysch unit is dominated by mudstones and siltstones interbedded with sandstones, gravels and pebbly mudstones [Shapiro and Petrina, 1985]. The flysch deposits locally are typical turbidite sequences. The spatial distribution of sandstone beds is not uniform; mudstones and siltstones compose in some areas sandstone-free members up to several hundreds meter thick. Structurally interleaved are (a) serpentinite blocks commonly interpreted as protrusions, and (b) volcanic blocks considered either as olistoliths or as tectonic outliers of an oceanic crust or as lenses conformable with the bedding. The latter are mainly pillow basalts with N- and E-MORB geochemistry [Kravchenko-Berezhnoy and Nazimova, 1991]. In this paper we also demonstrate that some mudstones spatially associated with N- and E-MORB basaltic olistoliths might be also considered as exotic blocks as well. The flysch deposits are either folded or form monoclines dipping to the northwest. The latter are separated by thrusts that are conformable with the bed dipping or are marked by protrusions or lenses of serpentinites.

The sandstones from the typical flysch unit are dated at the middle Eocene to early Oligocene (45.6 $\pm 4.6-29.9 \pm 3.6$ Ma, FT zircon single-grain ages) [Garver et al., 2001; Shapiro et al., 2004]. The paleontological ages available are controversial. The sediments contain benthic foraminifera of the upper Cretaceous - lower Paleocene [Shapiro and Petrina, 1985], mollusks of the Eocene (V. M. Gladikova determinations, see [Chekhovich et al., 1990]), and nannoplankton of the upper Cretaceous (Santonian-Campanian) [Shapiro and Petrina, 1985; Shapiro et al., 2004] and upper Eocene [Stcherbinina, 1997]. The upper Cretaceous nannoplankton species are thought to be redeposited [Shapiro et al., 2004]. But it should be noted that the upper Cretaceous nannoplankton species were found in the shales associated with the exotic basaltic blocks.

Typical mélange includes a chaotic distribution of fragments and blocks of tuffs, cherts, basalts and more rarely limestones and sandstones, whose size varies from a few centimeters to a few meters, embedded in the fragmental matrix of argillites or silicic argillite. The mélange units also contain large undeformed blocks composed of bedded gravel to psammitic tuffs, emerald-green, wax-red, gray and black cherts, argillites. By some researchers, they are considered as exotic blocks tectonically juxtaposed with argillites [Chamov, 1996], which is consistent with the shale geochemistry indicating their formation in the oceanic setting. The large blocks composed of pillow volcanics, red jaspers and pink limestones occur more rarely, and they resemble olistostromes [Shapiro and Petrina, 1985]. The volcanics are represented by tholeiitic basalts and subordinate amount of low-K andesites and calc-alkaline basaltic andesites spatially associate with tuffs [Chekhovich et al., 1990].

The timing of the mélange formation is accepted to be the Late Oligocene - Early Miocene based on the FT zircon single-grain age determinations $(26.1 \pm 1.5-18.6 \pm 3.5 \mathrm{Ma})$ in sandstones [Garver et al., 2001; Shapiro et al., 2004]. The findings of the upper Cretaceous and upper Paleogene nannoplankton species in the single samples were interpreted as the result of the shale redeposition [Shapiro et al., 2004]. The radiolarian ages of cherts in the mélange correspond to the upper Eocene [Chekhovich et al., 1990].

Relationships between the units. The relationships of the flysch with adjacent structural units are most disputed. The flysch and volcanic-sedimentary complexes are spatially separated by the bedded sequence of argillites and gravel, psephitic and psammitic tuffites and tuffs; and this sequence was recognized as the single structural unit referred to as the transitional volcanic-sedimentary. The tuffites and tuffs of the latter mainly consist of angular and rounded debris of plagioclase, clinopyroxene, pseudomorphs of iddingsite and magnetite after olivine, aphiric, Pl-porphiric and glomeroporhiric basalts, basaltic dolerites, and subordinate amount of argillites and cherts, e.g. material of the volcanic-sedimentary and volcanic-siliceous complexes. The shales interbedded with these tuffites and tuffs show the derivation from the differentiated continental or island arc. The tuff from these sequence is dated at $50.2 \pm 3.2 \mathrm{Ma}$ (FT zircon single-grain age determinations) [Garver et al., 2001; Shapiro et al., 2004]. The paleontological age of these shales range in the interval of the middle to lower Eocene, and these shales contain the redeposited upper Cretaceous nannoplankton species [Shapiro et al., 2004]. According to [Shapiro and Petrina, 1985; Shapiro et al., 2004], the mélange thrust over the flysch deposits and composes the core of the regional antiform with the hinge dipping to the south. The flysch represents its limbs. The southeastern limb of the anticline is complicated by numerous tectonic fractures.

Occurrence of cobbles and boulders. The cobbles and boulders occur in both flysch and mélange units. They are commonly disseminated in the mudstones and siltstones and are locally concentrated in the relatively thin layers. About $50 \%$ of all cobbles are dacites, rhyodacites, rhyolites, their tuffs, ignimbrites and their intrusive equivalents [Shapiro et al., 2000]. The total amount of intermediate and basic volcanics and intrusive rocks does not exceed $20 \%$. About $25 \%$ of all cobbles are gray and black recrystallized chert, and the remainder of cobbles are tuffites and polymictic sandstones. The predominance among the cobbles of the subaerial felsic volcanics, their tuffs and ignimbrites indicates that a prove- 
nance source region was above the sea level by the time of its formation.

\section{Sampling and Methods}

The studied sediments were collected along a $\sim 20 \mathrm{~km}-$ long transect covering the transitional volcanic-sedimentary, flysch and sedimentary mélange units. Volcanic and plutonic cobbles were sampled from pebbly mudstone beds in the flysch and mélange units (Figure 2). Chemical analysis were obtained for 25 shale and 10 granite/rhyolite samples; the fission-track ages were calculated for four samples of igneous rock cobbles.

The major elements were determined by X-ray fluorescence at the United Institute of Geology, Geophysics and Mineralogy, Siberian Branch of Russian Academy of Sciences, Novosibirsk, Russia using standard procedures for controlling accuracy and reproducibility of the analysis. The trace elements were analyzed using an Inductively Coupled Plasma Mass Spectrometer (PerkinElmer/SciexElan 6100 DRC) at Geology Department, Union College, Schenectady, NY, USA. Sample preparation was done using a low-pressure HF digestion (see http://www.union.edu/PUBLIC/ GEODEPT/hollocher/icp-ms/). The reported trace-element concentrations represent the average of duplicates. The relative deviation of mean values of duplicates is $5-15 \%$ for REE and $1-5 \%$ for other elements. The major and trace-element analyses obtained for shales and igneous cobbles are given in Tables $1 \mathrm{a}-1 \mathrm{c}$ and 2 , respectively.

For fission-track (FT) dating of zircon, standard techniques were followed [Garver et al., 2000]. For each sample, twenty to fifty zircons were counted using the external detector method with a zeta calibration. Neutron fluence during irradiation was estimated using glass dosimeters $(\mathrm{CN}-5)$ and zeta factor was calculated by repeatedly measuring standards of known age and the fluence for standards of known age. For this study, a zeta value of $362.22 \pm 8.88$ was determined based on measurements from nine samples (seven Fish Canyon Tuff, two Buluk Tuff) in three in different irradiation runs (U14, U15, U19) carried out over two years at the Oregon State nuclear reactor. Each fission-track grain age distribution was deconvolved into a set of components using the best-fit binomial peak fitting routine [Brandon, 1992, 1996; Brandon and Vance, 1992; Garver et al., 2000]. Calculations for populations of grain ages were done using the BINOMFIT program [Brandon, 1991, unpublished]. The results for the FT zircon ages are recorded in Tables 3 and 4 .

\section{Shales: Geochemical Data and Their Interpretation}

Despite the rather dramatic lithologic and structural differences between the units, shale from the flysch and sedimentary mélange as well as shales collected next to basaltic blocks of N- and E-MORB types and those from the "exotic" blocks show only slight compositional variations (Tables 1a$1 \mathrm{c}$ and $5 \mathrm{a}-5 \mathrm{c})$. This important finding suggests that these sediments may be derived from the same or a very similar provenance whereas the minor difference between the shales collected in different geological positions might be attributed to different conditions and/or tectonic settings of sedimentation.

\section{Mineral Phases Controlling Element Distribution}

The studied sediments are characterized by normal alkali $\left(\mathrm{K}_{2} \mathrm{O} / \mathrm{Al}_{2} \mathrm{O}_{3}=0.13-0.17\right)$ and iron $\left(\mathrm{Fe}_{2} \mathrm{O}_{3}+\mathrm{MgO} / \mathrm{SiO}_{2}=\right.$ 0.11-0.17) indexes suggesting the presence of feldspar and mafic minerals only in minor amount. The shales show moderately strong to strong correlation between $\mathrm{TiO}_{2}$ and $\mathrm{Al}_{2} \mathrm{O}_{3}$, and between these elements and $\mathrm{SiO}_{2}$ (Table 6) indicating that their contents were mainly controlled by silica and clay minerals. The correlation between $\mathrm{K}_{2} \mathrm{O}$ and $\mathrm{SiO}_{2}$ is moderate to moderately strong (Table 6), and the $\mathrm{K}_{2} \mathrm{O} / \mathrm{Al}_{2} \mathrm{O}_{3}$ ratio values ranging in the interval of $0.13-0.17$ suggest that shales contain hydromica and chlorite whereas plagioclase either absent or present only in a subordinate amount [Yudovich, 1981]. $\mathrm{Fe}_{2} \mathrm{O}_{3}+\mathrm{MgO}$ exhibit poor correlation with $\mathrm{Al}_{2} \mathrm{O}_{3}$ and moderate correlation with $\mathrm{SiO}_{2}$ (Table 6), and this indicates partial control by mafic minerals and accessory oxides.

\section{Weathering}

The studied shales are characterized by moderate CIA $\left(\mathrm{Al}_{2} \mathrm{O}_{3} /\left(\mathrm{Al}_{2} \mathrm{O}_{3}+\mathrm{CaO}+\mathrm{Na}_{2} \mathrm{O}+\mathrm{K}_{2} \mathrm{O}\right)=73-80\right)$ index values suggesting that these rocks were affected by weathering. This is consistent with the presence of the Ce anomaly $\left(\mathrm{Ce} / \mathrm{Ce}^{*}=0.75-0.88\right)$, increasing of $\mathrm{Th} / \mathrm{U}$ ratios with increasing of Th (Figure 3 ) and moderate Th and U correlation (Table 6). The $\mathrm{Rb} / \mathrm{Sr}(0.04-1.29)$ ratios are widely variable. As the $\mathrm{Sr}$ and $\mathrm{Na}_{2} \mathrm{O}$ show the clear correlation, and neither Sr nor $\mathrm{Na}_{2} \mathrm{O}$ correlate with $\mathrm{SiO}_{2}$ or $\mathrm{Al}_{2} \mathrm{O}_{3}$ (Table 6); the sea-water - rock interaction is assumed.

\section{Geochemical Indicators of Detrital Component}

There is no doubt that the studied sediments were originally dominated by detrital component as they are: (a) abundant in $\mathrm{Al}_{2} \mathrm{O}_{3}$ [Dymond, 1981], (b) phyllosilicates controlling $\mathrm{Al}_{2} \mathrm{O}_{3}$ abundance commonly resulted from low temperature and pressure metamorphic transformations of mafic silicate minerals, and (b) $\mathrm{Fe}_{2} \mathrm{O}_{3}+\mathrm{MgO}$ concentrations are controlled by mafic minerals and accessory oxides. Nonetheless, as deposition of shales took place under water, and the studied rocks are moderately weathered and experienced sea-water - rock interaction, it is very important to identify elements whose behavior was controlled by detrital components providing the most valuable information on the studied sediment provenance and tectonic setting. Recall that several sources (or components) might contribute to sediments. 
Table 1a. Contents of major and trace elements in shales of the transitional volcanic-sedimentary unit, northeastern Karaginski Island

\begin{tabular}{|c|c|c|c|c|c|c|c|c|}
\hline Sample & $00-1 \mathrm{X}$ & $00-1 \mathrm{U}$ & $00-1 \mathrm{R}$ & $00-1 \mathrm{M}$ & $00-1 \mathrm{I}$ & $00-1 \mathrm{G}$ & $00-1 \mathrm{D}$ & $00-1 \mathrm{~A}$ \\
\hline \multicolumn{9}{|c|}{ The transitional volcanic-sedimentary unit } \\
\hline $\mathrm{SiO}_{2}$ & 60.68 & 61.25 & 62.74 & 64.99 & 64.04 & 62.07 & 62.58 & 63.21 \\
\hline $\mathrm{TiO}_{2}$ & 0.90 & 0.84 & 0.74 & 0.72 & 0.72 & 0.81 & 0.80 & 0.75 \\
\hline $\mathrm{Al}_{2} \mathrm{O}_{3}$ & 16.64 & 16.96 & 15.47 & 14.70 & 14.52 & 15.81 & 15.84 & 15.51 \\
\hline $\mathrm{Fe}_{2} \mathrm{O}_{3}$ & 7.14 & 6.97 & 7.65 & 6.96 & 7.31 & 7.14 & 7.13 & 7.56 \\
\hline $\mathrm{MnO}$ & 0.13 & 0.12 & 0.13 & 0.12 & 0.13 & 0.14 & 0.12 & 0.12 \\
\hline $\mathrm{MgO}$ & 2.64 & 2.50 & 2.48 & 2.46 & 2.52 & 2.50 & 2.31 & 2.30 \\
\hline $\mathrm{CaO}$ & 1.04 & 1.01 & 1.28 & 0.92 & 1.38 & 0.92 & 0.93 & 0.85 \\
\hline $\mathrm{Na}_{2} \mathrm{O}$ & 1.96 & 2.33 & 2.47 & 2.26 & 1.85 & 1.87 & 1.93 & 2.52 \\
\hline $\mathrm{K}_{2} \mathrm{O}$ & 2.71 & 2.59 & 2.01 & 2.06 & 2.12 & 2.49 & 2.55 & 2.07 \\
\hline $\mathrm{P}_{2} \mathrm{O}_{5}$ & 0.13 & 0.14 & 0.15 & 0.13 & 0.12 & 0.18 & 0.11 & 0.14 \\
\hline Total & 93.97 & 94.71 & 95.12 & 95.32 & 94.71 & 93.93 & 94.30 & 95.03 \\
\hline LOI & 6.01 & 5.38 & 4.91 & 4.70 & 5.26 & 6.09 & 5.85 & 5.03 \\
\hline & \multicolumn{8}{|c|}{ ICP-MS data } \\
\hline $\mathrm{Sc}$ & 21.58 & 19.75 & 17.76 & 15.37 & 16.31 & 17.94 & 19.37 & 14.99 \\
\hline $\mathrm{V}$ & 195.9 & 178.9 & 155.6 & 135.3 & 141.1 & 173.1 & 178.7 & 145.1 \\
\hline $\mathrm{Co}$ & 10.04 & 10.16 & 18.85 & 14.90 & 19.18 & 10.66 & 10.38 & 16.03 \\
\hline $\mathrm{Ni}$ & 29.81 & 28.99 & 35.52 & 43.02 & 33.58 & 29.28 & 32.05 & 32.85 \\
\hline $\mathrm{Cr}$ & 77.4 & 77.6 & 79.2 & 83.6 & 69.6 & 75.4 & 77.6 & 82.6 \\
\hline $\mathrm{Rb}$ & 92.70 & 93.37 & 69.75 & 71.91 & 79.14 & 87.44 & 94.20 & 59.44 \\
\hline $\mathrm{Sr}$ & 151 & 195 & 210 & 181 & 158 & 136 & 146 & 193 \\
\hline $\mathrm{Y}$ & 25 & 27 & 22 & 31 & 22 & 25 & 24 & 21 \\
\hline $\mathrm{Zr}$ & 146 & 156 & 110 & 114 & 120 & 130 & 134 & 114 \\
\hline $\mathrm{Nb}$ & 9.96 & 10.60 & 8.28 & 8.47 & 8.95 & 9.36 & 9.51 & 8.30 \\
\hline $\mathrm{Ba}$ & 606 & 693 & 679 & 617 & 554 & 556 & 617 & 973 \\
\hline $\mathrm{La}$ & 21.58 & 24.33 & 22.83 & 28.34 & 22.35 & 28.50 & 26.19 & 21.81 \\
\hline $\mathrm{Ce}$ & 39.57 & 45.36 & 43.36 & 49.50 & 43.26 & 49.51 & 44.30 & 40.66 \\
\hline $\mathrm{Pr}$ & 6.36 & 7.03 & 6.94 & 8.72 & 6.92 & 8.29 & 7.24 & 6.60 \\
\hline $\mathrm{Nd}$ & 24.53 & 27.73 & 26.40 & 34.98 & 27.01 & 31.99 & 27.00 & 25.29 \\
\hline $\mathrm{Sm}$ & 5.49 & 5.95 & 5.64 & 7.66 & 5.72 & 6.56 & 5.67 & 5.29 \\
\hline $\mathrm{Eu}$ & 1.19 & 1.29 & 1.21 & 1.57 & 1.12 & 1.35 & 1.13 & 1.09 \\
\hline $\mathrm{Gd}$ & 4.91 & 5.38 & 4.75 & 7.05 & 4.75 & 5.65 & 4.85 & 4.60 \\
\hline $\mathrm{Tb}$ & 0.78 & 0.84 & 0.72 & 0.99 & 0.68 & 0.84 & 0.75 & 0.70 \\
\hline Dy & 4.85 & 5.28 & 4.26 & 5.60 & 4.11 & 5.18 & 4.66 & 4.25 \\
\hline Ho & 1.03 & 1.08 & 0.85 & 1.10 & 0.84 & 1.04 & 0.94 & 0.83 \\
\hline $\mathrm{Er}$ & 2.87 & 2.98 & 2.32 & 2.87 & 2.34 & 2.88 & 2.61 & 2.29 \\
\hline $\mathrm{Tm}$ & 0.44 & 0.45 & 0.34 & 0.41 & 0.36 & 0.44 & 0.40 & 0.34 \\
\hline $\mathrm{Yb}$ & 2.93 & 3.01 & 2.28 & 2.62 & 2.41 & 2.81 & 2.59 & 2.20 \\
\hline $\mathrm{Lu}$ & 0.46 & 0.47 & 0.36 & 0.42 & 0.38 & 0.45 & 0.41 & 0.34 \\
\hline Hf & 4.36 & 4.65 & 3.20 & 3.08 & 3.28 & 3.81 & 3.91 & 3.25 \\
\hline $\mathrm{Ta}$ & 0.56 & 0.57 & 0.43 & 0.44 & 0.45 & 0.54 & 0.53 & 0.46 \\
\hline Th & 8.27 & 9.41 & 6.56 & 6.59 & 6.94 & 7.90 & 8.04 & 6.76 \\
\hline \multirow[t]{2}{*}{$\mathrm{U}$} & 2.51 & 2.78 & 2.09 & 2.05 & 2.42 & 2.53 & 2.39 & 2.08 \\
\hline & \multicolumn{8}{|c|}{ XRF data } \\
\hline $\mathrm{Cu}$ & 76.00 & 66.00 & 50.00 & 45.00 & 41.00 & 47.00 & - & 37.00 \\
\hline $\mathrm{Zn}$ & 106.00 & 116.00 & 95.00 & 141.00 & 105.00 & 109.00 & - & 100.00 \\
\hline
\end{tabular}


Table 1b. Contents of major and trace elements in shales of the flysch unit, northeastern Karaginski Island

\begin{tabular}{|c|c|c|c|c|c|c|c|c|c|c|}
\hline Sample & KASH-1 & KASH-3 & KASH-6 & KASH-12 & KASH-13 & KASH-14 & KASH-16 & KASH-8 & KASH-10 & KASH-15 \\
\hline & \multicolumn{10}{|c|}{ The flysch unit (accretionary wedge complex) } \\
\hline & \multicolumn{7}{|c|}{ matrix } & \multicolumn{3}{|c|}{ "exotic" blocks } \\
\hline $\mathrm{SiO}_{2}$ & 66.44 & 65.50 & 65.98 & 67.36 & 67.35 & 63.41 & 65.22 & 63.11 & 62.59 & 65.61 \\
\hline $\mathrm{TiO}_{2}$ & 0.72 & 0.77 & 0.74 & 0.70 & 0.72 & 0.84 & 0.73 & 0.77 & 0.80 & 0.74 \\
\hline $\mathrm{Al}_{2} \mathrm{O}_{3}$ & 13.98 & 15.04 & 14.50 & 13.63 & 14.01 & 15.70 & 15.01 & 15.21 & 15.57 & 15.08 \\
\hline $\mathrm{Fe}_{2} \mathrm{O}_{3}$ & 6.62 & 6.47 & 6.67 & 6.24 & 6.56 & 6.98 & 6.18 & 7.34 & 7.10 & 6.42 \\
\hline $\mathrm{MnO}$ & 0.14 & 0.13 & 0.14 & 0.13 & 0.13 & 0.13 & 0.13 & 0.34 & 0.35 & 0.16 \\
\hline $\mathrm{MgO}$ & 2.60 & 2.66 & 2.65 & 2.63 & 2.44 & 2.70 & 2.66 & 3.14 & 3.15 & 2.41 \\
\hline $\mathrm{CaO}$ & 0.71 & 0.74 & 0.59 & 0.87 & 0.33 & 0.87 & 0.91 & 0.86 & 1.06 & 0.58 \\
\hline $\mathrm{Na}_{2} \mathrm{O}$ & 1.88 & 2.05 & 2.07 & 1.78 & 2.09 & 1.97 & 1.86 & 1.77 & 1.69 & 1.94 \\
\hline $\mathrm{K}_{2} \mathrm{O}$ & 2.08 & 1.96 & 1.90 & 1.91 & 1.81 & 2.14 & 2.50 & 2.27 & 2.50 & 2.20 \\
\hline $\mathrm{P}_{2} \mathrm{O}_{5}$ & 0.17 & 0.11 & 0.12 & 0.10 & 0.10 & 0.13 & 0.12 & 0.08 & 0.08 & 0.11 \\
\hline Total & 95.34 & 95.43 & 95.36 & 95.35 & 95.54 & 94.87 & 95.32 & 94.89 & 94.89 & 95.25 \\
\hline \multirow[t]{2}{*}{ LOI } & 4.70 & 4.61 & 4.64 & 4.66 & 4.48 & 5.08 & 4.84 & 5.09 & 5.09 & 4.88 \\
\hline & \multicolumn{10}{|c|}{ ICP-MS data } \\
\hline $\mathrm{Sc}$ & 16.66 & 18.15 & 17.32 & 16.61 & 18.64 & 19.99 & 16.88 & 20.09 & 21.40 & 17.35 \\
\hline $\mathrm{V}$ & 136.2 & 170.5 & 157.3 & 146.8 & 160.7 & 179.2 & 141.1 & 190.1 & 206.2 & 164.5 \\
\hline $\mathrm{Co}$ & 11.81 & 11.86 & 12.09 & 12.21 & 13.67 & 14.35 & 13.09 & 22.83 & 23.15 & 13.66 \\
\hline $\mathrm{Ni}$ & 29.00 & 37.45 & 32.81 & 30.84 & 30.83 & 35.02 & 26.77 & 42.70 & 65.76 & 38.14 \\
\hline $\mathrm{Cr}$ & 79.2 & 92.5 & 91.5 & 79.9 & 74.9 & 95.4 & 75.6 & 54.5 & 62.4 & 81.7 \\
\hline $\mathrm{Rb}$ & 74.11 & 76.96 & 66.28 & 69.34 & 65.80 & 78.71 & 87.53 & 79.80 & 92.59 & 78.56 \\
\hline $\mathrm{Sr}$ & 164 & 138 & 154 & 142 & 166 & 128 & 128 & 114 & 108 & 108 \\
\hline $\mathrm{Y}$ & 22 & 23 & 20 & 19 & 22 & 25 & 22 & 25 & 28 & 21 \\
\hline $\mathrm{Zr}$ & 96 & 107 & 99 & 104 & 103 & 118 & 123 & 128 & 137 & 112 \\
\hline $\mathrm{Nb}$ & 7.72 & 8.83 & 7.55 & 7.50 & 6.91 & 9.52 & 7.43 & 7.71 & 8.83 & 8.19 \\
\hline $\mathrm{Ba}$ & 605 & 542 & 607 & 475 & 555 & 472 & 462 & 894 & 871 & 588 \\
\hline $\mathrm{La}$ & 23.28 & 23.54 & 18.98 & 18.45 & 20.26 & 22.68 & 17.91 & 24.44 & 26.91 & 20.43 \\
\hline $\mathrm{Ce}$ & 40.89 & 39.93 & 33.07 & 34.88 & 33.80 & 41.90 & 35.41 & 46.89 & 50.08 & 36.22 \\
\hline $\mathrm{Pr}$ & 6.46 & 6.90 & 5.39 & 5.38 & 5.56 & 6.77 & 5.61 & 7.14 & 7.86 & 5.93 \\
\hline $\mathrm{Nd}$ & 23.92 & 25.65 & 20.83 & 20.08 & 21.92 & 26.70 & 22.05 & 27.75 & 30.32 & 22.67 \\
\hline $\mathrm{Sm}$ & 4.88 & 5.54 & 4.38 & 4.13 & 4.65 & 5.57 & 4.67 & 6.00 & 6.42 & 4.77 \\
\hline $\mathrm{Eu}$ & 1.04 & 1.18 & 0.94 & 0.72 & 0.97 & 1.20 & 0.97 & 1.11 & 1.22 & 0.94 \\
\hline $\mathrm{Gd}$ & 4.22 & 4.81 & 3.93 & 3.46 & 4.17 & 4.77 & 4.06 & 5.16 & 5.54 & 4.21 \\
\hline $\mathrm{Tb}$ & 0.61 & 0.71 & 0.59 & 0.54 & 0.63 & 0.74 & 0.62 & 0.77 & 0.84 & 0.63 \\
\hline Dy & 3.83 & 4.36 & 3.73 & 3.46 & 3.92 & 4.68 & 3.87 & 4.86 & 5.29 & 4.01 \\
\hline Ho & 0.78 & 0.89 & 0.77 & 0.72 & 0.79 & 0.94 & 0.80 & 1.02 & 1.04 & 0.83 \\
\hline $\mathrm{Er}$ & 2.12 & 2.39 & 2.11 & 2.01 & 2.14 & 2.60 & 2.17 & 2.82 & 2.88 & 2.29 \\
\hline $\mathrm{Tm}$ & 0.33 & 0.36 & 0.32 & 0.32 & 0.33 & 0.40 & 0.33 & 0.44 & 0.44 & 0.35 \\
\hline $\mathrm{Yb}$ & 2.19 & 2.35 & 2.13 & 2.14 & 2.21 & 2.64 & 2.23 & 2.97 & 2.95 & 2.33 \\
\hline $\mathrm{Lu}$ & 0.35 & 0.36 & 0.34 & 0.34 & 0.36 & 0.42 & 0.34 & 0.47 & 0.48 & 0.38 \\
\hline $\mathrm{Hf}$ & 2.65 & 3.20 & 2.86 & 2.90 & 2.95 & 3.48 & 3.17 & 3.64 & 3.76 & 3.24 \\
\hline $\mathrm{Ta}$ & 0.39 & 0.46 & 0.40 & 0.39 & 0.35 & 0.49 & 0.38 & 0.41 & 0.44 & 0.44 \\
\hline $\mathrm{Th}$ & 6.40 & 6.17 & 5.46 & 6.20 & 5.56 & 7.23 & 6.07 & 6.52 & 7.50 & 6.66 \\
\hline \multirow[t]{2}{*}{$\mathrm{U}$} & 2.28 & 2.09 & 2.13 & 2.41 & 2.50 & 2.67 & 2.28 & 2.28 & 2.46 & 2.59 \\
\hline & \multicolumn{10}{|c|}{$\mathrm{XRF}$ data } \\
\hline $\mathrm{Cu}$ & 181.00 & 51.00 & 58.00 & 51.00 & 58.00 & 52.00 & 52.00 & 264.00 & 83.00 & 56.00 \\
\hline $\mathrm{Zn}$ & 94.00 & 109.00 & 89.00 & 100.00 & 96.00 & 102.00 & 96.00 & 118.00 & 164.00 & 109.00 \\
\hline
\end{tabular}


Table 1c. Contents of major and trace elements in shales of the sedimentary mélange unit, northeastern Karaginski Island

\begin{tabular}{|c|c|c|c|c|c|c|c|}
\hline Sample & KASH-24 & KASH-26 & KASH-28 & KASH-20 & KASH-22 & KASH-30 & KASH-32 \\
\hline & \multicolumn{7}{|c|}{ The sedimentary mélange unit (accretionary wedge complex) } \\
\hline & \multicolumn{3}{|c|}{ matrix } & \multicolumn{4}{|c|}{ "exotic" blocks } \\
\hline $\mathrm{SiO}_{2}$ & 64.68 & 63.42 & 64.45 & 65.60 & 72.17 & 66.89 & 69.71 \\
\hline $\mathrm{TiO}_{2}$ & 0.80 & 0.77 & 0.81 & 0.68 & 0.58 & 0.31 & 0.27 \\
\hline $\mathrm{Al}_{2} \mathrm{O}_{3}$ & 15.84 & 15.55 & 15.79 & 13.66 & 11.77 & 14.57 & 13.14 \\
\hline $\mathrm{Fe}_{2} \mathrm{O}_{3}$ & 6.50 & 6.94 & 6.55 & 6.72 & 5.54 & 4.78 & 5.91 \\
\hline $\mathrm{MnO}$ & 0.12 & 0.12 & 0.12 & 0.38 & 0.20 & 0.61 & 0.13 \\
\hline $\mathrm{MgO}$ & 2.40 & 2.51 & 2.46 & 3.95 & 2.13 & 3.03 & 2.28 \\
\hline $\mathrm{CaO}$ & 0.69 & 0.92 & 0.64 & 0.32 & 0.26 & 1.05 & 0.50 \\
\hline $\mathrm{Na}_{2} \mathrm{O}$ & 1.86 & 1.86 & 2.38 & 0.77 & 1.28 & 3.87 & 2.04 \\
\hline $\mathrm{K}_{2} \mathrm{O}$ & 2.22 & 2.50 & 2.12 & 2.33 & 1.59 & 1.17 & 1.62 \\
\hline $\mathrm{P}_{2} \mathrm{O}_{5}$ & 0.14 & 0.12 & 0.14 & 0.08 & 0.07 & 0.08 & 0.11 \\
\hline Total & 95.25 & 94.71 & 95.46 & 94.49 & 95.59 & 96.36 & 95.71 \\
\hline \multirow[t]{2}{*}{ LOI } & 4.79 & 5.31 & 4.68 & 5.56 & 4.40 & 3.53 & 4.00 \\
\hline & \multicolumn{7}{|c|}{ ICP-MS data } \\
\hline $\mathrm{Sc}$ & 16.65 & 17.22 & 17.02 & 24.19 & 14.54 & 8.60 & 6.86 \\
\hline V & 154.1 & 158.9 & 149.6 & 168.2 & 131.5 & 54.3 & 43.3 \\
\hline $\mathrm{Co}$ & 11.10 & 9.46 & 11.84 & 34.46 & 21.94 & 35.32 & 6.41 \\
\hline $\mathrm{Ni}$ & 30.31 & 28.52 & 31.90 & 105.95 & 43.72 & 39.75 & 15.23 \\
\hline $\mathrm{Cr}$ & 89.0 & 72.7 & 89.7 & 53.3 & 74.1 & 13.7 & 29.1 \\
\hline $\mathrm{Rb}$ & 80.11 & 95.12 & 81.22 & 76.19 & 54.20 & 27.00 & 35.75 \\
\hline $\mathrm{Sr}$ & 116 & 140 & 125 & 59 & 92 & 700 & 168 \\
\hline $\mathrm{Y}$ & 22 & 25 & 24 & 28 & 18 & 35 & 24 \\
\hline $\mathrm{Zr}$ & 127 & 137 & 133 & 126 & 91 & 176 & 94 \\
\hline $\mathrm{Nb}$ & 9.34 & 9.34 & 10.12 & 5.81 & 5.66 & 3.88 & 1.84 \\
\hline $\mathrm{Ba}$ & 437 & 568 & 457 & 858 & 1102 & 6145 & 1759 \\
\hline $\mathrm{La}$ & 22.98 & 25.66 & 23.90 & 26.24 & 16.75 & 22.43 & 14.19 \\
\hline $\mathrm{Ce}$ & 41.29 & 47.30 & 41.01 & 54.38 & 32.06 & 37.27 & 25.41 \\
\hline $\mathrm{Pr}$ & 6.15 & 7.18 & 6.20 & 7.70 & 4.73 & 6.63 & 5.05 \\
\hline $\mathrm{Nd}$ & 23.15 & 27.23 & 24.29 & 30.21 & 18.03 & 27.46 & 22.42 \\
\hline $\mathrm{Sm}$ & 4.79 & 5.61 & 4.92 & 6.30 & 3.68 & 3.96 & 4.55 \\
\hline $\mathrm{Eu}$ & 0.98 & 1.09 & 0.91 & 1.30 & 0.65 & 0.21 & 0.76 \\
\hline $\mathrm{Gd}$ & 4.08 & 4.65 & 4.29 & 5.54 & 3.51 & 6.69 & 4.82 \\
\hline $\mathrm{Tb}$ & 0.63 & 0.71 & 0.66 & 0.81 & 0.52 & 0.92 & 0.68 \\
\hline Dy & 3.94 & 4.51 & 4.13 & 5.14 & 3.27 & 5.48 & 4.19 \\
\hline Но & 0.81 & 0.91 & 0.86 & 1.04 & 0.65 & 1.08 & 0.83 \\
\hline Er & 2.31 & 2.53 & 2.42 & 2.88 & 1.81 & 2.86 & 2.25 \\
\hline $\mathrm{Tm}$ & 0.35 & 0.39 & 0.37 & 0.46 & 0.28 & 0.40 & 0.32 \\
\hline $\mathrm{Yb}$ & 2.38 & 2.63 & 2.45 & 3.22 & 1.98 & 2.53 & 2.05 \\
\hline $\mathrm{Lu}$ & 0.37 & 0.41 & 0.38 & 0.52 & 0.31 & 0.40 & 0.31 \\
\hline Hf & 3.42 & 3.74 & 3.55 & 3.19 & 2.40 & 4.65 & 2.67 \\
\hline $\mathrm{Ta}$ & 0.49 & 0.50 & 0.51 & 0.29 & 0.30 & 0.20 & 0.08 \\
\hline Th & 6.77 & 7.72 & 7.45 & 7.50 & 4.63 & 15.71 & 2.03 \\
\hline \multirow[t]{2}{*}{$\mathrm{U}$} & 2.32 & 2.31 & 2.41 & 2.49 & 2.88 & 3.14 & 0.85 \\
\hline & \multicolumn{7}{|c|}{$\mathrm{XRF}$ data } \\
\hline $\mathrm{Cu}$ & 28.00 & 54.00 & 40.00 & 163.00 & 69.00 & 196.00 & 35.00 \\
\hline $\mathrm{Zn}$ & 91.00 & 101.00 & 91.00 & 170.00 & 120.00 & 99.00 & 79.00 \\
\hline
\end{tabular}


Table 2. Contents of major and trace elements in felsic clasts in sedimentary units, Karaginski Island

\begin{tabular}{|c|c|c|c|c|c|c|c|c|c|c|}
\hline $\begin{array}{l}\text { Sample } \\
\text { No }\end{array}$ & \multicolumn{4}{|c|}{ KA-21-R2 KA-21G KA-21-TB KA-21A } & KA-21A-R8 & KA-21R & \multicolumn{2}{|c|}{ KA-29C KA-21-R13 } & \multirow{2}{*}{$\begin{array}{c}\text { KA-23 } \\
\mathrm{G}\end{array}$} & \multirow{2}{*}{$\frac{\mathrm{KA}-29 \mathrm{~B}}{\mathrm{G}}$} \\
\hline $\begin{array}{l}\text { Rock } \\
\text { type }\end{array}$ & $\operatorname{Tr}$ & $\mathrm{T}$ & $\mathrm{Rd}$ & $\mathrm{R}$ & $\operatorname{Tr}$ & $\operatorname{Tr}$ & G & G & & \\
\hline Series & \multicolumn{4}{|c|}{ Low-K tonalite-trodhjemite series } & \multicolumn{2}{|c|}{$\begin{array}{l}\text { Med-K tonalite-trodhjemite } \\
\text { series }\end{array}$} & \multicolumn{4}{|c|}{ High-K calc-alkaline series } \\
\hline $\mathrm{SiO}_{2}$ & 71.83 & 69.07 & 70.66 & 73.52 & 76.04 & 78.03 & 77.04 & 73.82 & 75.87 & 71.61 \\
\hline $\mathrm{TiO}_{2}$ & 0.19 & 0.64 & 0.54 & 0.55 & 0.23 & 0.17 & 0.18 & 0.22 & 0.15 & 0.33 \\
\hline $\mathrm{Al}_{2} \mathrm{O}_{3}$ & 16.23 & 15.57 & 15.08 & 10.60 & 12.45 & 11.36 & 12.16 & 13.74 & 13.52 & 14.11 \\
\hline $\mathrm{Fe}_{2} \mathrm{O}_{3}$ & 1.59 & 4.13 & 3.71 & 4.27 & 2.86 & 2.80 & 1.83 & 2.59 & 1.49 & 2.86 \\
\hline $\mathrm{MnO}$ & 0.12 & 0.13 & 0.14 & 0.19 & 0.12 & 0.12 & 0.12 & 0.12 & 0,11 & 0.13 \\
\hline $\mathrm{MgO}$ & 0.49 & 1.40 & 1.13 & 2.29 & 0.32 & 0.54 & 0.40 & 0.48 & 0.12 & 1.06 \\
\hline $\mathrm{CaO}$ & 2.87 & 1.53 & 0.97 & 2.01 & 0.29 & 0.20 & 1.09 & 0.67 & 0.44 & 1.73 \\
\hline $\mathrm{Na}_{2} \mathrm{O}$ & 4.32 & 4.92 & 5.15 & 3.13 & 4.61 & 3.71 & 3.29 & 3.53 & 3.58 & 3.03 \\
\hline $\mathrm{K}_{2} \mathrm{O}$ & 0.58 & 0.51 & 0.67 & 0.18 & 1.68 & 1.66 & 2.66 & 3.19 & 3.96 & 3.13 \\
\hline $\mathrm{P}_{2} \mathrm{O}_{5}$ & 0.09 & 0.12 & 0.14 & 0.14 & 0.05 & 0.03 & 0.04 & 0.06 & 0.04 & 0.07 \\
\hline Total & 98.31 & 98.02 & 98.19 & 96.88 & 98.65 & 98.62 & 98.81 & 98.42 & 99.28 & 98.06 \\
\hline LOI & 1.66 & 1.96 & 1.80 & 3.12 & 1.34 & 1.40 & 1.16 & 1.58 & 0.71 & 1.89 \\
\hline ASI & 1.25 & 1.36 & 1.38 & 1.18 & 1.25 & 1.37 & 1.18 & 1.31 & 1.23 & 1.23 \\
\hline & \multicolumn{10}{|c|}{ ICP-MS data } \\
\hline $\mathrm{Sc}, \mathrm{ppm}$ & 2.67 & 7.61 & 8.91 & 11.49 & 2.03 & 2.46 & 2.30 & 4.46 & 3.45 & 9.54 \\
\hline $\mathrm{V}$ & 8.76 & 46.70 & 16.26 & 23.31 & 3.51 & 4.34 & 4.69 & 7.36 & 2.96 & 22.11 \\
\hline $\mathrm{Co}$ & 4.80 & 11.10 & 6.04 & 6.18 & 3.13 & 2.02 & 2.19 & 7.61 & 0.92 & 4.78 \\
\hline $\mathrm{Ni}$ & 23.53 & 15.82 & 29.11 & 24.32 & 17.77 & 18.54 & 10.64 & 38.04 & 7.03 & 17.94 \\
\hline $\mathrm{Cr}$ & 31.95 & 11.93 & 37.13 & 15.37 & 18.81 & 18.02 & 12.80 & 24.19 & 16.12 & 32.74 \\
\hline $\mathrm{Rb}$ & 6.54 & 6.58 & 18.98 & 2.04 & 51.32 & 45.48 & 87.60 & 74.90 & 111.58 & 82.07 \\
\hline $\mathrm{Sr}$ & 312 & 237 & 80 & 58 & 167 & 104 & 96 & 92 & 55 & 167 \\
\hline $\mathrm{Y}$ & 3 & 14 & 14 & 14 & 48 & 39 & 22 & 10 & 15 & 22 \\
\hline $\mathrm{Zr}$ & 5 & 43 & 216 & 79 & 302 & 232 & 56 & 76 & 67 & 53 \\
\hline $\mathrm{Nb}$ & 1.29 & 7.20 & 10.30 & 1.47 & 23.38 & 28.79 & 14.74 & 8.57 & 12.74 & 6.88 \\
\hline $\mathrm{Ba}$ & 335 & 183 & 623 & 330 & 1309 & 2134 & 266 & 977 & 568 & 386 \\
\hline $\mathrm{La}$ & 6.47 & 17.49 & 20.22 & 4.29 & 90.11 & 49.75 & 35.87 & 29.31 & 35.50 & 25.52 \\
\hline $\mathrm{Ce}$ & 15.02 & 35.25 & 48.29 & 10.25 & 156.90 & 106.77 & 70.11 & 58.07 & 75.43 & 54.31 \\
\hline $\operatorname{Pr}$ & 1.82 & 4.55 & 5.49 & 1.53 & 24.18 & 13.08 & 7.99 & 6.92 & 8.71 & 6.57 \\
\hline $\mathrm{Nd}$ & 7.67 & 18.84 & 21.64 & 7.35 & 92.22 & 49.45 & 28.63 & 24.44 & 30.85 & 25.37 \\
\hline $\mathrm{Sm}$ & 1.19 & 3.47 & 3.52 & 1.72 & 16.70 & 7.99 & 4.13 & 3.57 & 4.17 & 4.25 \\
\hline $\mathrm{Eu}$ & 0.56 & 0.99 & 0.88 & 0.51 & 0.80 & 0.51 & 0.38 & 0.53 & 0.42 & 0.54 \\
\hline $\mathrm{Gd}$ & 1.04 & 2.92 & 2.88 & 2.10 & 14.24 & 6.59 & 3.31 & 2.54 & 2.99 & 3.82 \\
\hline $\mathrm{Tb}$ & 0.13 & 0.42 & 0.42 & 0.34 & 1.94 & 1.02 & 0.55 & 0.36 & 0.48 & 0.61 \\
\hline Dy & 0.62 & 2.56 & 2.52 & 2.33 & 10.94 & 6.59 & 3.68 & 2.13 & 3.05 & 3.85 \\
\hline Ho & 0.10 & 0.50 & 0.54 & 0.51 & 2.02 & 1.48 & 0.76 & 0.42 & 0.59 & 0.78 \\
\hline $\mathrm{Er}$ & 0.23 & 1.35 & 1.64 & 1.49 & 5.40 & 4.61 & 2.22 & 1.19 & 1.64 & 2.16 \\
\hline $\mathrm{Tm}$ & 0.03 & 0.20 & 0.26 & 0.24 & 0.83 & 0.79 & 0.36 & 0.20 & 0.26 & 0.32 \\
\hline $\mathrm{Yb}$ & 0.20 & 1.25 & 1.88 & 1.69 & 5.60 & 5.62 & 2.49 & 1.36 & 1.75 & 2.16 \\
\hline $\mathrm{Lu}$ & 0.03 & 0.20 & 0.32 & 0.29 & 0.91 & 0.92 & 0.39 & 0.22 & 0.27 & 0.35 \\
\hline $\mathrm{Hf}$ & 1.85 & 1.33 & 5.72 & 2.55 & 9.28 & 8.65 & 2.39 & 2.67 & 2.74 & 2.09 \\
\hline $\mathrm{Ta}$ & 0.07 & 0.31 & 0.50 & 0.07 & 1.30 & 1.53 & 1.18 & 0.61 & 0.81 & 0.48 \\
\hline $\mathrm{Pb}$ & 2.92 & 7.64 & 5.33 & 6.24 & 23.88 & 12.45 & 14.47 & 15.57 & 13.24 & 9.22 \\
\hline $\mathrm{Th}$ & 0.84 & 2.27 & 5.30 & 1.11 & 14.74 & 14.97 & 15.88 & 9.71 & 12.32 & 7.97 \\
\hline $\mathrm{U}$ & 0.69 & 0.99 & 3.23 & 1.37 & 5.59 & 8.22 & 2.58 & 3.90 & 2.58 & 2.30 \\
\hline
\end{tabular}

Note: $\mathrm{Tr}$ - trondhjemite, $\mathrm{T}$ - tonalite, $\mathrm{G}$ - granite, $\mathrm{Rd}$ - rhyodacite, $\mathrm{R}$ - rhyolite. ASI $=\mathrm{Al}_{2} \mathrm{O}_{3} /\left(\mathrm{CaO}+\mathrm{Na}_{2} \mathrm{O}+\mathrm{K}_{2} \mathrm{O}\right)_{\text {molar }}$. 

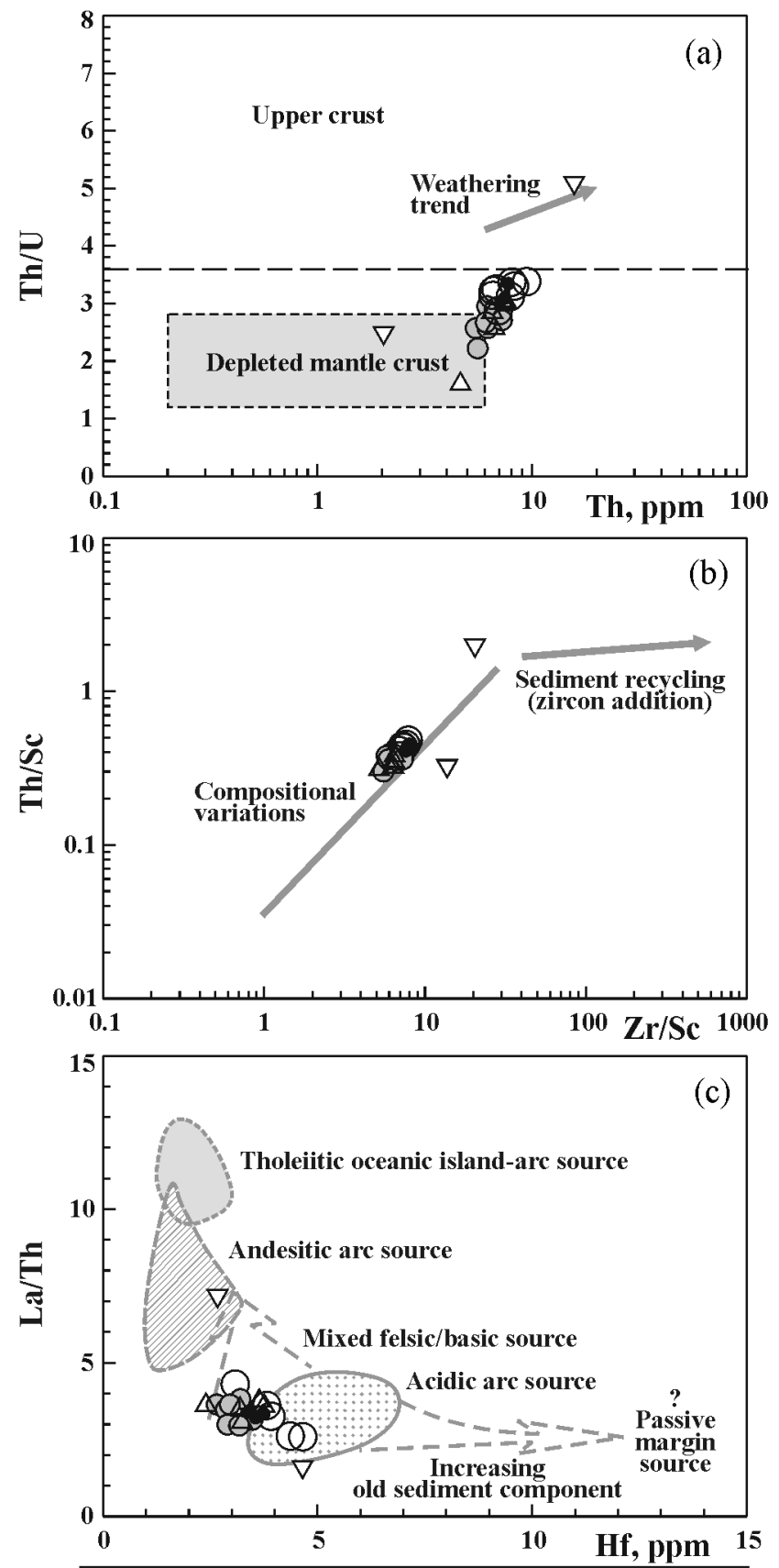

Shales from the transitional volcanic-sedimentary unit
Shales from the flysch unit
- Shales from the melange unit
$\Delta$ Shales associated with basaltic olistoliths of
N-MORB and E-MORB types in the flysch unit
$\nabla \begin{aligned} & \text { Shales from the undeformed "exotic" blocks } \\ & \text { with tuffs and cherts in the melange unit }\end{aligned}$

Figure 3. Th vs. Th/U (a), Zr/Sc vs. Th/Sc (b) plots (both after [McLennan et al., 1993]) and Hf vs. La/Th (c) (after [Floyd and Leveridge, 1987]) for the shales of northeastern Karaginski Island.
These sources are as follows: (a) detrital, (b) hydrothermal, (c) biogenic, (d) hydrogenous, and (e) dissolution residue [Dymond, 1981].

Application of the normative composition model (Table 7) shows that the studied rocks are dominated by detrital and biogenic components whereas the contribution of hydrothermal, hydrogeneous and dissoluble residue sources is negligible. As the biogenic source is assumed to be predominantly composed of opal and organic matter [Dymond, 1981], its addition to shales in northeastern Karaginski Island will be simply resulted in increasing of $\mathrm{SiO}_{2}$ contents in sediments relative to those in the provenance deposits and, therefore, in dilution of the detrital component but will not affect elemental ratios. The negligible or no contribution of material from hydrothermal, hydrogenous and dissoluble residue sources allows the suggestion that most of the elements in the studied rocks reflect their distribution in the provenance. The only exclusions are: (a) $\mathrm{Na}_{2} \mathrm{O}$ and $\mathrm{Sr}$, whose concentrations were modified owing the sea-water - rock interaction, (b) U and $\mathrm{Ce}$, whose contents were changed during the weathering; and (c) Ba, whose abnormally high abundance in a few samples is probably resulted from contribution of dissoluble residue component.

\section{Provenance and Tectonic Settings}

Geochemical features of the shales collected from the matrix of the transitional volcanic-sedimentary, flysch and mélange units are fairly uniform; and these rocks are considered together. In comparison to PAAS (the Post-Archean Australian Average Shale) whose composition is though to be parallel to the average composition of the upper continental crust [Taylor and McLennan, 1985], all these shales from northeastern Karaginski Island show the similar abundance in high-field strength elements (HFSE) and middle and heavy rear-earth elements (REE), but they are lower in large-ion lithophile elements (LILE), light REE and transitional metals (Figures 4 and 5a). These features indicate that the shales are composed of mainly upper continental crust material. The compositional difference between the studied rocks and PAAS can be attributed to different contribution of distinct provenance components such as: (a) old upper continental crust, (b) recycled sedimentary rocks, (c) young undifferentiated arc, (d) young differentiated arc, and (e) exotic components [McLennan et al., 1993].

By the evolved major element composition $(\mathrm{Si} / \mathrm{Al}=6.13$ 8.39; CIA $=73-77)$, high LREE abundance $\left((\mathrm{La} / \mathrm{Yb})_{\mathrm{N}}=\right.$ 4.09-6.02), pronounced negative $\mathrm{Eu}$ anomaly $\left(\mathrm{Eu} / \mathrm{Eu}^{*}=\right.$ $0.57-0.70<1)$ (Figure 5c), low Th/Sc $(0.31-0.48 \ll 1)$ and $\mathrm{La} / \mathrm{Sc}(1.00-1.59 \ll 4)$ ratios, the studied sediments resemble those deposited at young differentiated continental and island arcs [Garver and Scott, 1995; Taylor and McLennan, 1985]. This is consistent with the multi-elements patterns indicating that the shale relative abundance in LILE in comparison to HFSE, their depletion in $\mathrm{Nb}\left(\mathrm{Nb} / \mathrm{Nb}^{*}\right.$ $=0.15-0.27), \mathrm{Ta}\left(\mathrm{Ta} / \mathrm{Ta}^{*}=0.13-0.25\right)$ and $\mathrm{Ti}\left(\mathrm{Ti} / \mathrm{Ti}^{*}=\right.$ 0.35-0.65) is similar to those in intermediate to felsic vol- 
Table 3. Zircon FT peak ages for clasts in sedimentary units, Karaginski Island

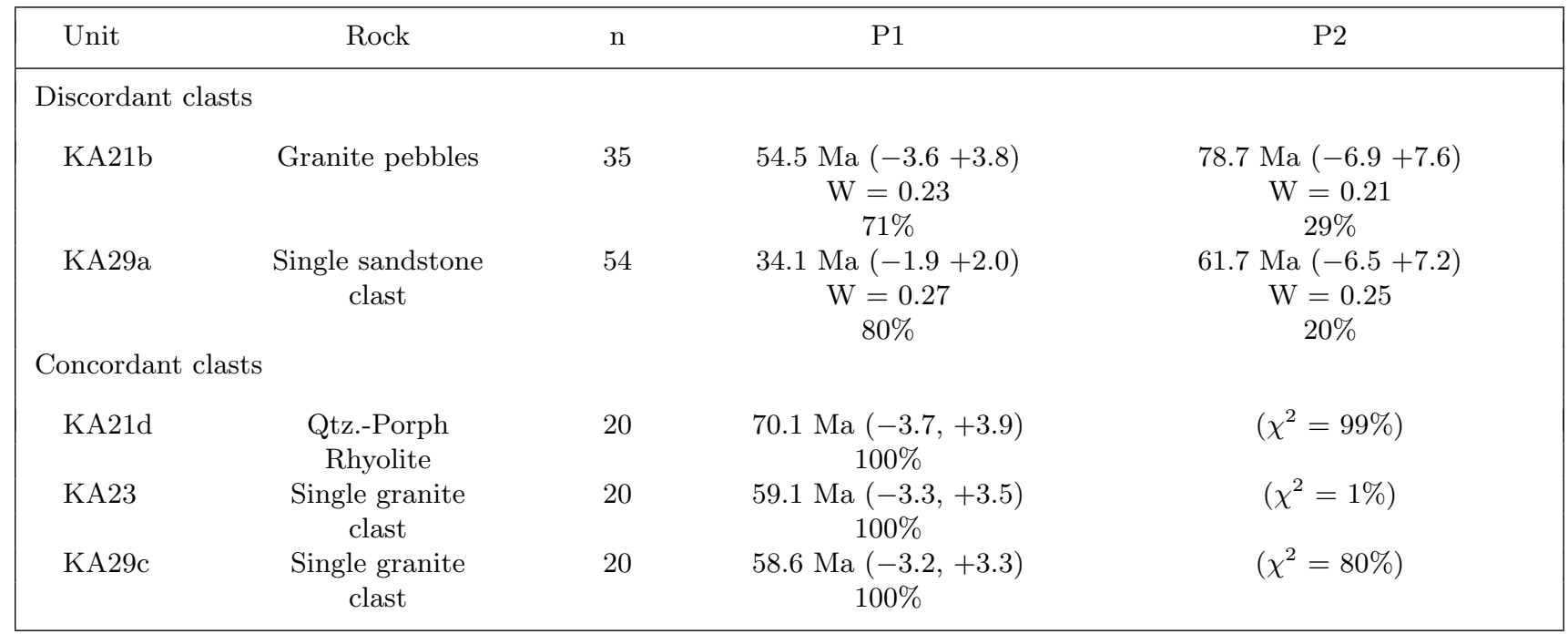

Note: Grain ages were determined using the standard techniques for the external detector method of detrital zircon (see [Garver and Brandon, 1994; Garver et al., 1999; Naeser, 1979]). All binomial peakfit ages give at 1 sigma. Zircons from single samples were imbedded into three separate $2 \times 2 \mathrm{~cm}$ PFA Teflon mounts at $330^{\circ} \mathrm{C}$. These mounts were then polished using 800 grit wet sandpaper, and then polished using $3 \mu \mathrm{m}, 1 \mu \mathrm{m}$ diamond paste and then finished using a $0.3 \mu \mathrm{m} \mathrm{Al}_{2} \mathrm{O}_{3}$ slurry. Each mount was etched different times to optimize countable grains (see [Garver et al., 1999; Naeser et al., 1987]). Etching was done in non-reactive Teflon dishes at $230^{\circ} \pm 2^{\circ} \mathrm{C}$ in a thermostatically controlled laboratory oven, but temperature was monitored using an external thermometer. Mounts were continuously etched for 15, 30 and $60 \mathrm{hrs}$ in a NaOH:KOH eutectic mixed with a ratio of 11.5:8.2 ([Gleadow et al., 1976]). Etched mounts were cleaned in dilute HF for 15 minutes, rinsed, dried, and then freshly cleaved, low-uranium mica flakes were affixed to the mounts. The samples were then irradiated at the Oregon State Nuclear Reactor with a nominal fleunce of $2 \times 10^{15}$ neutrons/cm ${ }^{2}$. Uranium-doped glass standards $(\mathrm{CN}-1)$ were placed at either end of the sample stack to monitor fleunce during irradiation, and from these glasses, a fleunce gradient was calculated and extrapolated values were used in age calculations. Twenty-five random but countable grains in a traverse were counted for each of six mounts and the results were combined for this analysis. Samples were counted using an automated stage fitted to a Zeiss Axioplan microscope with a magnification of $1250 \times(50 \times$ dry objective, $10 \times$ oculars, $2.5 \times$ tube factor). We used the zeta calibration approach in calculating ages with a zeta factor of $133.58 \pm 1.1$ (see [Wagner and van den Haute, 1992], for further details). Peak ages were calculated using a binomial statistical analysis (see [Brandon, 1996]). The number of grains in each analysis is represented by "n".

Table 4. Zircon Fission-track data, all clasts, Karaginski Island

\begin{tabular}{|llccccccccccccc}
\hline Sample rock & $\rho_{s}$ & $N_{s}$ & $\rho_{i}$ & $N_{i}$ & $\rho_{d}$ & $N_{d}$ & n & $\chi^{2}$ & Age & $-1 \sigma$ & $+1 \sigma$ & U \pm 2 se \\
\hline Concordant clasts & & & & & & & & & & \\
KA21d & Rhyolite & $4.86 \times 10^{6}$ & 1278 & $3.63 \times 10^{6}$ & 955 & $2.91 \times 10^{5}$ & 1002 & 20 & 99 & 70.1 & -3.7 & +3.9 & $153.4 \pm 12.1$ \\
KA23 & Granite & $5.47 \times 10^{6}$ & 965 & $4.90 \times 10^{6}$ & 865 & $2.94 \times 10^{5}$ & 1012 & 20 & 1.2 & 59.1 & -3.3 & +3.5 & $205.2 \pm 16.8$ \\
KA29c & Granite & $1.02 \times 10^{7}$ & 1058 & $9.52 \times 10^{6}$ & 985 & $3.03 \times 10^{5}$ & 1043 & 20 & 80 & 58.6 & -3.2 & +3.3 & $386.5 \pm 30.4$ \\
& & & & & & & & & & & & \\
Discordant clasts & & & & & & & & & & & \\
KA21b & Granite Pbls & $1.03 \times 10^{7}$ & 2114 & $8.60 \times 10^{6}$ & 1760 & $2.82 \times 10^{5}$ & 971 & 35 & 0.1 & 60.4 & -3.4 & +3.6 & $371.2 \pm 24.6$ \\
KA29a & Sandstone & $4.05 \times 10^{6}$ & 1835 & $5.65 \times 10^{6}$ & 2559 & $2.96 \times 10^{5}$ & 1027 & 54 & 0.0 & 38.5 & -2.1 & +2.2 & $231.5 \pm 14.0$ \\
\hline
\end{tabular}

Note: In this table, $\rho_{s}$ is the density $\left(\mathrm{cm}^{2}\right)$ of spontaneous tracks, and $N_{s}$ is the number of spontaneous tracks counted; $\rho_{i}$ is the density $\left(\mathrm{cm}^{2}\right)$ of induced tracks; and $\rho_{d}$ is the density $\left(\mathrm{cm}^{2}\right)$ of tracks on the fleunce monitor (CN5); " $\mathrm{n}$ " is the number of grains counted; and $\chi^{2}$ is the Chi-squared probability(\%). Zircon Fission-track ages $( \pm 1 \sigma)$ were determined using the Zeta method, and ages were calculated using the computer program and equations in Brandon [1992]. A Zeta factor of 362.22 \pm 8.88 ( \pm 1 se) is based on 9 determinations from both the Fish Canyon Tuff and the Buluk tuff. All ages with $\chi^{2}>1 \%$ are reported as pooled ages, otherwise, central ages are shown. Glass monitors (CN-5), placed at the top and bottom of the irradiation package were used to determine the fluence gradient. All samples were counted at $1250^{\mathrm{x}}$ using a dry $100^{\mathrm{x}}$ objective $\left(10^{\mathrm{x}}\right.$ oculars and $1.25^{\mathrm{x}}$ tube factor $)$ on an Olympus BMAX 60 microscope fitted with an automated stage and a Calcomp digitizing tablet. 
Table 5a. Indicative ratios for shales of the transitional volcanic--sedimentary unit, northeastern Karaginski Island

\begin{tabular}{|c|c|c|c|c|c|c|c|c|}
\hline Sample & $00-1 \mathrm{X}$ & $00-1 \mathrm{U}$ & $00-1 \mathrm{R}$ & $00-1 \mathrm{M}$ & $00-1 \mathrm{I}$ & $00-1 \mathrm{G}$ & 00-1D & $00-1 \mathrm{~A}$ \\
\hline $\mathrm{Si} / \mathrm{Al}^{*}$ & 5.58 & 5.53 & 5.93 & 5.99 & 6.07 & 5.75 & 5.76 & 5.94 \\
\hline $\begin{array}{l}\mathrm{K}_{2} \mathrm{O} / \mathrm{Al}_{2} \mathrm{O}_{3} \\
\left(\mathrm{Fe}_{2} \mathrm{O}_{3}+\mathrm{MgO}\right) /\end{array}$ & 0.16 & 0.15 & 0.13 & 0.14 & 0.15 & 0.16 & 0.16 & 0.13 \\
\hline $\mathrm{SiO}_{2}$ & 0.16 & 0.15 & 0.16 & 0.14 & 0.15 & 0.16 & 0.15 & 0.16 \\
\hline $\mathrm{Al}_{2} \mathrm{O}_{3} / \mathrm{SiO}_{2}$ & 0.27 & 0.28 & 0.25 & 0.23 & 0.23 & 0.25 & 0.25 & 0.25 \\
\hline CIA & 67.40 & 66.69 & 64.36 & 65.86 & 64.92 & 67.99 & 67.50 & 66.16 \\
\hline $\mathrm{Fe} / \mathrm{Mn}$ & 49.60 & 52.46 & 53.15 & 52.38 & 50.79 & 46.06 & 53.66 & 56.90 \\
\hline$(\mathrm{La} / \mathrm{Yb})_{\mathrm{N}}$ & 5.28 & 5.80 & 7.19 & 7.76 & 6.64 & 7.28 & 7.26 & 7.12 \\
\hline $\mathrm{Eu} / \mathrm{Eu}^{*}$ & 0.69 & 0.69 & 0.71 & 0.65 & 0.65 & 0.67 & 0.66 & 0.67 \\
\hline $\mathrm{Ce} / \mathrm{Ce}^{*}$ & 0.98 & 1.00 & 1.00 & 0.91 & 1.01 & 0.93 & 0.93 & 0.98 \\
\hline $\mathrm{Th} / \mathrm{U}$ & 3.30 & 3.38 & 3.14 & 3.22 & 2.87 & 3.12 & 3.37 & 3.25 \\
\hline $\mathrm{Th} / \mathrm{Sc}$ & 0.38 & 0.48 & 0.37 & 0.43 & 0.43 & 0.44 & 0.41 & 0.45 \\
\hline $\mathrm{La} / \mathrm{Sc}$ & 1.00 & 1.23 & 1.29 & 1.84 & 1.37 & 1.59 & 1.35 & 1.46 \\
\hline $\mathrm{Rb} / \mathrm{Sr}$ & 0.62 & 0.48 & 0.33 & 0.40 & 0.50 & 0.64 & 0.65 & 0.31 \\
\hline $\mathrm{Cr} / \mathrm{Ni}$ & 2.60 & 2.68 & 2.23 & 1.94 & 2.07 & 2.57 & 2.42 & 2.51 \\
\hline $\mathrm{Nb} / \mathrm{Nb}^{*}$ & 0.27 & 0.25 & 0.24 & 0.22 & 0.26 & 0.23 & 0.24 & 0.25 \\
\hline $\mathrm{Ta} / \mathrm{Ta}^{*}$ & 0.26 & 0.24 & 0.23 & 0.21 & 0.23 & 0.23 & 0.23 & 0.24 \\
\hline $\mathrm{Ti} / \mathrm{Ti}^{*}$ & 0.59 & 0.50 & 0.51 & 0.35 & 0.51 & 0.48 & 0.54 & 0.53 \\
\hline
\end{tabular}

Table 5b. Indicative ratios for shales of the flysch unit, northeastern Karaginski Island

\begin{tabular}{|c|c|c|c|c|c|c|c|c|c|c|}
\hline Sample & KASH-1 & KASH-3 & KASH-6 & KASH-12 & KASH-13 & KASH-14 & KASH-16 & KASH-8 & KASH-10 & KASH-15 \\
\hline & \multicolumn{7}{|c|}{ matrix } & \multicolumn{3}{|c|}{ "exotic" blocks } \\
\hline $\mathrm{Si} / \mathrm{Al}^{*}$ & 6.08 & 5.83 & 5.98 & 6.07 & 6.07 & 5.76 & 5.77 & 5.95 & 5.82 & 5.82 \\
\hline $\begin{array}{l}\mathrm{K}_{2} \mathrm{O} / \mathrm{Al}_{2} \mathrm{O}_{3} \\
\left(\mathrm{Fe}_{2} \mathrm{O}_{3}+\mathrm{MgO}\right) /\end{array}$ & 0.15 & 0.13 & 0.13 & 0.14 & 0.13 & 0.14 & 0.17 & 0.15 & 0.16 & 0.15 \\
\hline $\mathrm{SiO}_{2}$ & 0.14 & 0.14 & 0.14 & 0.13 & 0.13 & 0.15 & 0.14 & 0.17 & 0.16 & 0.13 \\
\hline $\mathrm{Al}_{2} \mathrm{O}_{3} / \mathrm{SiO}_{2}$ & 0.21 & 0.23 & 0.22 & 0.20 & 0.21 & 0.25 & 0.23 & 0.24 & 0.25 & 0.23 \\
\hline CIA & 67.81 & 68.74 & 68.93 & 67.45 & 70.02 & 68.74 & 66.92 & 68.69 & 67.74 & 69.47 \\
\hline $\mathrm{Fe} / \mathrm{Mn}$ & 42.71 & 44.95 & 43.03 & 43.35 & 45.58 & 48.49 & 42.94 & 19.50 & 18.32 & 36.24 \\
\hline$(\mathrm{La} / \mathrm{Yb})_{\mathrm{N}}$ & 7.63 & 7.20 & 6.39 & 6.17 & 6.57 & 6.17 & 5.76 & 5.90 & 6.54 & 6.29 \\
\hline $\mathrm{Eu} / \mathrm{Eu}^{*}$ & 0.70 & 0.69 & 0.68 & 0.58 & 0.67 & 0.71 & 0.68 & 0.60 & 0.62 & 0.64 \\
\hline $\mathrm{Ce} / \mathrm{Ce}^{*}$ & 0.91 & 0.95 & 1.03 & 0.92 & 0.98 & 0.95 & 1.11 & 1.00 & 1.01 & 1.02 \\
\hline $\mathrm{Th} / \mathrm{U}$ & 2.81 & 2.95 & 2.57 & 2.57 & 2.22 & 2.71 & 2.67 & 2.85 & 3.05 & 2.57 \\
\hline $\mathrm{Th} / \mathrm{Sc}$ & 0.38 & 0.34 & 0.32 & 0.37 & 0.30 & 0.36 & 0.36 & 0.32 & 0.35 & 0.38 \\
\hline $\mathrm{La} / \mathrm{Sc}$ & 1.40 & 1.30 & 1.10 & 1.11 & 1.09 & 1.13 & 1.06 & 1.22 & 1.26 & 1.18 \\
\hline $\mathrm{Rb} / \mathrm{Sr}$ & 0.45 & 0.56 & 0.43 & 0.49 & 0.40 & 0.62 & 0.68 & 0.70 & 0.85 & 0.73 \\
\hline $\mathrm{Cr} / \mathrm{Ni}$ & 2.73 & 2.47 & 2.79 & 2.59 & 2.43 & 2.72 & 2.82 & 1.28 & 0.95 & 2.14 \\
\hline $\mathrm{Nb} / \mathrm{Nb}^{*}$ & 0.23 & 0.27 & 0.27 & 0.25 & 0.24 & 0.27 & 0.26 & 0.22 & 0.22 & 0.25 \\
\hline $\mathrm{Ta} / \mathrm{Ta}^{*}$ & 0.20 & 0.24 & 0.25 & 0.23 & 0.21 & 0.25 & 0.23 & 0.21 & 0.20 & 0.24 \\
\hline $\mathrm{Ti} / \mathrm{Ti}^{*}$ & 0.57 & 0.53 & 0.62 & 0.65 & 0.56 & 0.57 & 0.58 & 0.49 & 0.47 & 0.58 \\
\hline
\end{tabular}

Note: (a) $\mathrm{Si} / \mathrm{Al}^{*}$ values are reculculated to biogenic-free residue, and (b) $\mathrm{CIA}=\mathrm{Al}_{2} \mathrm{O}_{3} /\left(\mathrm{Al}_{2} \mathrm{O}_{3}+\mathrm{CaO}+\mathrm{Na} 2 \mathrm{O}+\mathrm{K} 2 \mathrm{O}\right)$. 
Table 5c. Indicative ratios for shales of the sedimentary mélange unit, northeastern Karaginski Island

\begin{tabular}{|c|c|c|c|c|c|c|c|}
\hline Sample & KASH-24 & KASH-26 & KASH-28 & KASh-20 & KASH-22 & KASH-30 & KASH-32 \\
\hline & \multicolumn{3}{|c|}{ matrix } & \multicolumn{4}{|c|}{ "exotic" blocks } \\
\hline $\mathrm{Si} / \mathrm{Al}^{*}$ & 5.68 & 5.79 & 5.69 & 6.17 & 6.44 & 5.47 & 6.17 \\
\hline $\begin{array}{l}\mathrm{K}_{2} \mathrm{O} / \mathrm{Al}_{2} \mathrm{O}_{3} \\
\left(\mathrm{Fe}_{2} \mathrm{O}_{3}+\mathrm{MgO}\right) /\end{array}$ & 0.14 & 0.16 & 0.13 & 0.17 & 0.14 & 0.08 & 0.12 \\
\hline $\mathrm{SiO}_{2}$ & 0.14 & 0.15 & 0.14 & 0.16 & 0.11 & 0.12 & 0.12 \\
\hline $\mathrm{Al}_{2} \mathrm{O}_{3} / \mathrm{SiO}_{2}$ & 0.24 & 0.25 & 0.24 & 0.21 & 0.16 & 0.22 & 0.19 \\
\hline CIA & 70.22 & 67.64 & 68.17 & 75.76 & 73.24 & 60.43 & 68.59 \\
\hline $\mathrm{Fe} / \mathrm{Mn}$ & 48.92 & 52.23 & 49.30 & 15.97 & 25.02 & 3.48 & 20.19 \\
\hline$(\mathrm{La} / \mathrm{Yb})_{\mathrm{N}}$ & 6.92 & 6.99 & 7.00 & 5.84 & 6.07 & 6.36 & 4.96 \\
\hline $\mathrm{Eu} / \mathrm{Eu}^{*}$ & 0.67 & 0.65 & 0.60 & 0.67 & 0.55 & 0.12 & 0.49 \\
\hline $\mathrm{Ce} / \mathrm{Ce}^{*}$ & 0.97 & 0.88 & 0.87 & 1.01 & 1.01 & 0.62 & 0.61 \\
\hline $\mathrm{Th} / \mathrm{U}$ & 2.92 & 3.34 & 3.09 & 3.01 & 1.60 & 1.82 & 2.39 \\
\hline $\mathrm{Th} / \mathrm{Sc}$ & 0.41 & 0.45 & 0.44 & 0.31 & 0.32 & 0.66 & 0.30 \\
\hline $\mathrm{La} / \mathrm{Sc}$ & 1.38 & 1.49 & 1.40 & 1.08 & 1.15 & 2.61 & 2.07 \\
\hline $\mathrm{Rb} / \mathrm{Sr}$ & 0.69 & 0.68 & 0.65 & 1.29 & 0.59 & 0.04 & 0.21 \\
\hline $\mathrm{Cr} / \mathrm{Ni}$ & 2.94 & 2.55 & 2.81 & 0.50 & 1.70 & 0.35 & 1.91 \\
\hline $\mathrm{Nb} / \mathrm{Nb}^{*}$ & 0.27 & 0.24 & 0.27 & 0.15 & 0.23 & 0.07 & 0.12 \\
\hline $\mathrm{Ta} / \mathrm{Ta}^{*}$ & 0.25 & 0.22 & 0.24 & 0.13 & 0.22 & 0.07 & 0.09 \\
\hline $\mathrm{Ti} / \mathrm{Ti}^{*}$ & 0.64 & 0.54 & 0.61 & 0.41 & 0.54 & 0.16 & 0.19 \\
\hline
\end{tabular}

canics of calc-alkaline series (Figure 5b). The relatively high $\mathrm{Cr} / \mathrm{Ni}$ ratios ranging in the interval of $1.94-2.94$ are indicative of mainly volcanic source with perhaps negligible ultramafic rocks [Garver and Scott, 1995; Garver et al., 1996].

At the same time, the shales from the matrix of the accretionary wedge units are very uniform in composition that might be resulted from rock recycling or erosion of the old continental crust. The latter is highly unlikely as the geochemical features of the shales significantly differ from those typical of PAAS. The sedimentary rock recycling is commonly recognized from the heavy mineral concentrations such as $\mathrm{Zr}$ and Hf [McLennan et al., 1993]. Although the shales in Karaginski Island do not show chemical indicators of zircon addition (Figure $3 \mathrm{~b}$ ); the recycling most probably took place. In one of the shale samples collected from the mélange unit there were found nannoplankton species indicative of the Turonian-Santonian (upper Cretaceous) and Bartonian (upper Eocene) ages. This phenomenon was attributed to redeposition of the upper Cretaceous nannoplankton species as zircon from the sandstones collected from the same locality was dated at $26 \mathrm{Ma}$ by FT-method [Garver et al., 2001; Shapiro et al., 2004].

The shales spatially associate with the basaltic blocks with $N$ - and E-MORB chemistry in the flysch unit and those associate with the emerald-green beds in the mélange unit are very similar by most parameters to those from the matrix (Figures $3-5$, Tables $5 \mathrm{~b}$ and $5 \mathrm{c}$ ) suggesting the similar provenance for the sediments from all these geological positions. The only significant difference is the former abundances in $\mathrm{MnO}$ resulted in the much lower $\mathrm{Fe} / \mathrm{Mn}$ ratios. For the comparison, the $\mathrm{Fe} / \mathrm{Mn}$ ratios range in the intervals of $16-36<40$ in the shales associate with basaltic blocks and emerald-green cherts and $40<43-57<80$ in shales from the matrix of the studied units. As the hydrothermal source was not recognized from the studied shale chemistry (Table 7), the Fe/Mn ratios probably indicates the shales deposition in deep- and shallow-water conditions, respectively [Rozen et al, 1994]. The close spatial proximity of shales with the lowest Fe/Mn ratios to the basaltic bodies showing $\mathrm{N}$ - and E-MORB chemistry in the flysch unit allows us to suggest that (a) the accretionary wedge units may contain "exotic" sedimentary blocks (although they were not identified during the field studies), and (b) these sediments may be originally deposited in the oceanic/marginal sea setting. The elevated $\mathrm{Fe} / \mathrm{Mn}$ values in the shales from the matrix of the accretionary wedge units may be indicative of their deposition in a forearc basin or oceanic/marginal sea highs.

Although the shales composing matrix of the accretionary wedge units, on the one hand, and those associated with the basaltic blocks of N- and E-MORB chemistry and emeraldgreen cherts, on the other hand, were suggested to be deposited in different geodynamic settings, their common compositional variations are easy to explain. The relative abundance of all shales in detrital and biogenic material (Table 7) is consistent with their deposition in the region closer than 1000-1500 km from the continent [Dymond, 1981]. The estimated amount of biogenic and detrital component inputs (Table 7) in shales from these geological positions are similar.

The shales from the "exotic" blocks in the mélange unit compositionally a little differ from the shales in other geological positions (Figures 3-5, Tables 5a-5c). In particular, they are characterized by elevated contents of $\mathrm{Ba}$ and $\mathrm{Sr}$ (Table 1c, Figure 5) and more pronounced negative anomalies of $\mathrm{Eu}\left(\mathrm{Eu} / \mathrm{Eu}^{*}=0.12-0.49\right), \mathrm{Ti}\left(\mathrm{Ti} / \mathrm{Ti}^{*}=0.16-0.19\right)$ and $\mathrm{Nb}\left(\mathrm{Nb} / \mathrm{Nb}^{*}=0.07-0.12\right)$. Nonetheless, the relative LILE/HFSE abundance (Figure 5) and the indicative elemental ratios in these shales ranging in the intervals of 7.79 

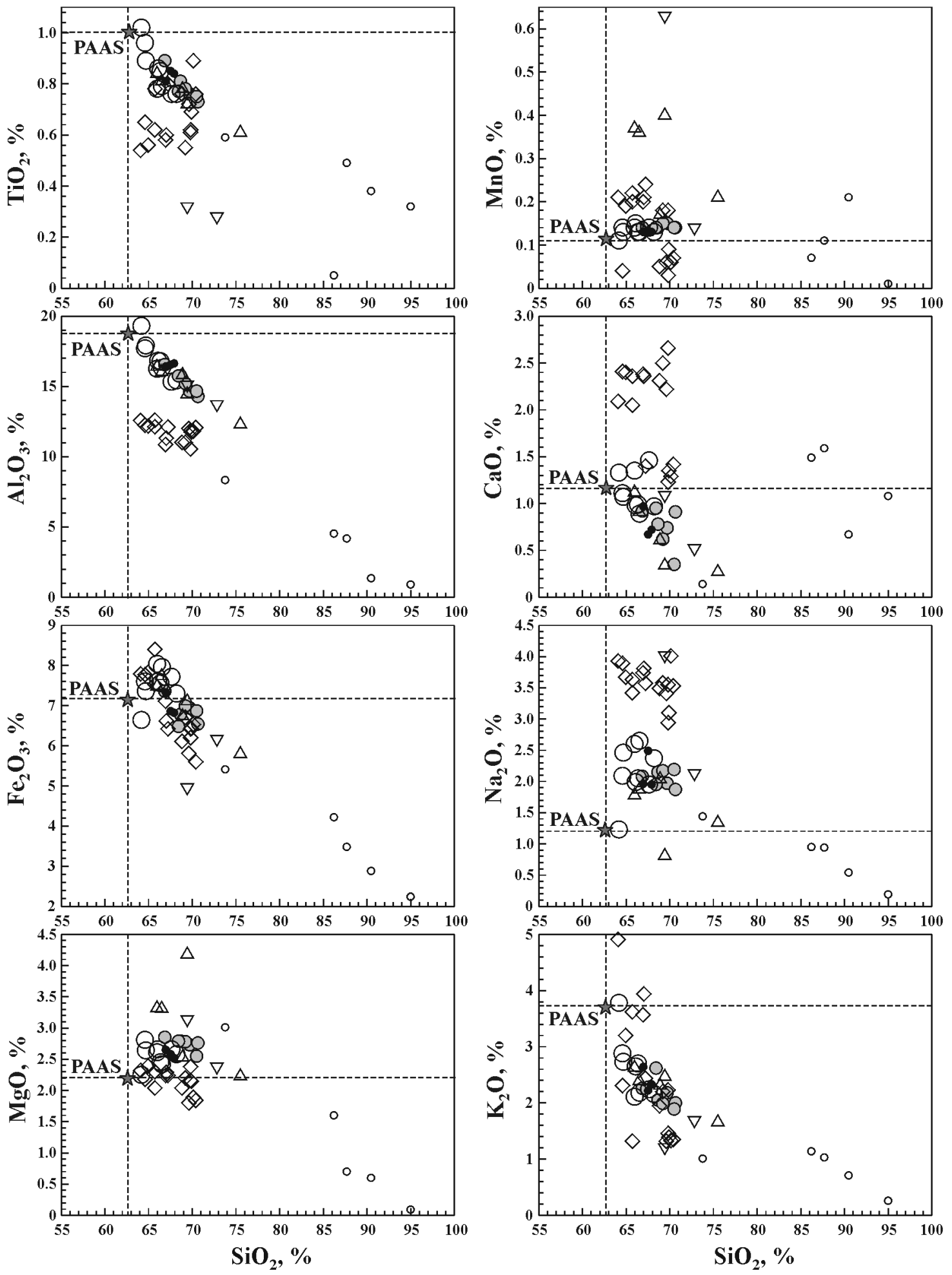

$\diamond$ Sandstones (original data from Chamov, 1996) - Silicites (original data from Chamov, 1996)

Figure 4. Variations of major elements as a function of $\mathrm{SiO}_{2}$ contents in the silicites, shales and sandstones of northeastern Karaginski Island. For symbols see also Figure 3. 

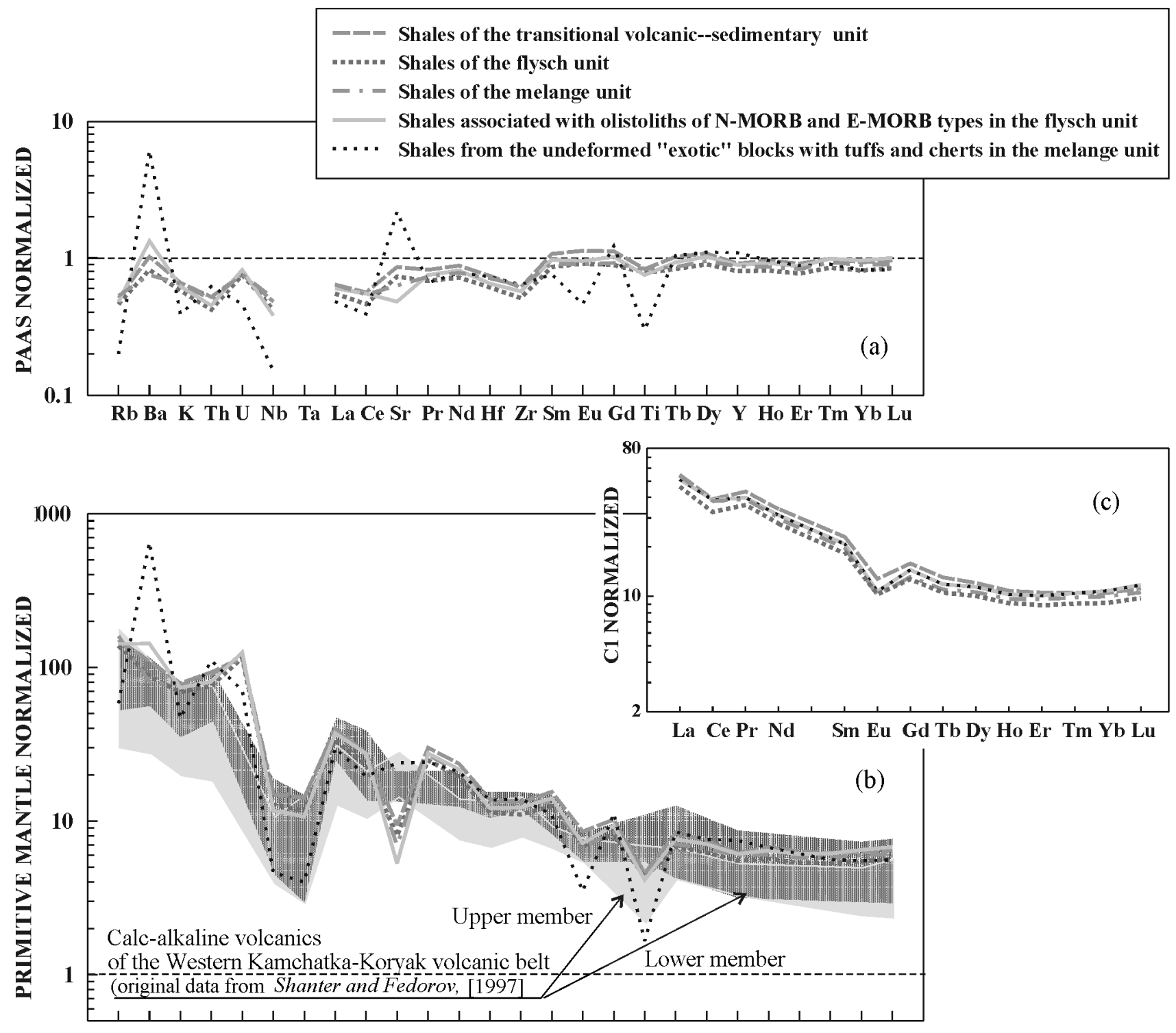

Rb Ba K Th U Nb Ta La Ce Sr Pr Nd Hf Zr Sm Eu Gd Ti Tb Dy Y Ho Er Tm Yb Lu

Figure 5. Normalized multi-element ( $\mathrm{a}$ and b) and REE (c) average spectra for the shales of northeastern Karaginski Island.

Normalizing data are from Taylor and McLennan [1985] for PAAS, from Hofmann [1988] for primitive mantle and from Anders and Grevesse [1989] for chondrite.

9.01 for $\mathrm{Si} / \mathrm{Al}$ (5.47-6.17 for $\left.\mathrm{Si}^{*} / \mathrm{Al}\right), 71-76$ for CIA, 3.854.93 for $(\mathrm{La} / \mathrm{Yb})_{\mathrm{N}}, 0.30-0.66 \ll 1$ for $\mathrm{Th} / \mathrm{Sc}, 2.07-2.61 \ll 4$ for $\mathrm{La} / \mathrm{Sc}$, and 1.82-2.39 for $\mathrm{Th} / \mathrm{U}$ suggest the main contribution of a differentiated continental and/or volcanic arc material as well. The specific geochemical features of the shales from the "exotic" blocks in the mélange unit might be partially attributed to the highest contribution of the biogenic component (Table 7) and/or to derivation of volcanic material from a different differentiated volcanic arc.

The Fe/Mn values in the shales of the "exotic" blocks are highly variable (7-41) but they are low to moderately low and most probably indicative of the shale deposition under deep-water and relatively deep-water conditions. Taken into account, the results of the normative composition model indicating the inputs of $29.35-31.44 \%$ and $68.35-69.02 \%$ of biogenic and detrital materials, respectively, combined with the fact of interbeddding of the shales with coarse-grained tuffs, it should be concluded that the deposition of shales from the "exotic" blocks occurred in close proximity to the coast, possibly, in the fore-arc basin. 
Table 6. Linear correlation coefficients for selected elements of the sediments on northeastern Karaginski Island

\begin{tabular}{|lcccc|}
\hline & transitional volcanic-sedimentary unit & flysch unit & mélange unit & all units \\
\hline $\mathrm{TiO}_{2}-\mathrm{Al}_{2} \mathrm{O}_{3}$ & 0.8416 & 0.8067 & 0.6168 & 0.7958 \\
$\mathrm{TiO}_{2}-\mathrm{SiO}_{2}$ & 0.7422 & 0.8223 & 0.6246 & 0.6053 \\
$\mathrm{Al}_{2} \mathrm{O}_{3}$ & 0.9111 & 0.9545 & 0.9999 & 0.8720 \\
$\mathrm{~K}_{2} \mathrm{O}-\mathrm{SiO}_{2}$ & 0.5245 & 0.3200 & 0.6044 & 0.5484 \\
$\left(\mathrm{Fe}_{2} \mathrm{O}_{3}+\mathrm{MgO}\right)-\mathrm{Al}_{2} \mathrm{O}_{3}$ & 0.0033 & 0.3754 & 0.9162 & 0.2107 \\
$\left(\mathrm{Fe}_{2} \mathrm{O}_{3}+\mathrm{MgO}\right)-\mathrm{SiO}_{2}$ & 0.0502 & 0.4836 & 0.9206 & 0.5221 \\
$\mathrm{Sr}-\mathrm{Na}_{2} \mathrm{O}$ & 0.8704 & 0.0918 & 0.0206 & 0.4660 \\
$\mathrm{Sr}-\mathrm{SiO}_{2}$ & 0.0004 & 0.5045 & 0.9961 & 0.0578 \\
$\mathrm{Na}_{2} \mathrm{O}-\mathrm{SiO}_{2}$ & 0.0065 & 0.0281 & 0.0066 & 0.1321 \\
$\mathrm{Sr}-\mathrm{Al}_{2} \mathrm{O}_{3}$ & 0.0110 & 0.5254 & 0.9950 & 0.0500 \\
$\mathrm{Na}_{2} \mathrm{O}-\mathrm{AL}_{2} \mathrm{O}_{3}$ & 0.6000 & 0.0817 & 0.0053 & 0.0799 \\
\hline
\end{tabular}

Table 7. Results of the normative analysis of the sediments from northeatern Karaginski Island

\begin{tabular}{|lcccccccc|}
\hline Sample & $00-1 \mathrm{X}$ & $00-1 \mathrm{U}$ & $00-1 \mathrm{R}$ & $00-1 \mathrm{M}$ & $00-1 \mathrm{I}$ & $00-1 \mathrm{G}$ & $00-1 \mathrm{D}$ & $00-1 \mathrm{~A}$ \\
\hline & \multicolumn{8}{c}{ transitional volcanic-sedimentary unit } \\
\hline Detrital & 90.19 & 90.13 & 86.09 & 79.75 & 81.00 & 86.27 & 85.84 & 85.79 \\
Hydrothermal & - & - & - & - & - & - & - & - \\
Biogenic & 9.81 & 9.88 & 13.91 & 20.25 & 19.00 & 13.73 & 14.16 & 14.21 \\
Authigenic & - & - & - & - & - & - & - & - \\
Residual & - & - & - & - & - & - & - \\
\hline
\end{tabular}

\begin{tabular}{|c|c|c|c|c|c|c|c|c|c|c|}
\hline Sample & KASH-1 & KASH-3 & KASH-6 & KASH-12 & KASH-13 & KASH-14 & KASH-16 & KASH-8 & KASH-10 & KASH-15 \\
\hline & \multicolumn{10}{|c|}{ flysch unit (accretionary wedge complex) } \\
\hline & \multicolumn{7}{|c|}{ matrix } & \multicolumn{3}{|c|}{ "exotic" blocks } \\
\hline Detrital & 75.37 & 78.85 & 77.44 & 72.35 & 74.43 & 84.06 & 78.15 & 84.39 & 85.30 & 78.78 \\
\hline Hydrothermal & - & - & - & - & - & - & - & - & - & - \\
\hline Biogenic & 24.63 & 21.15 & 22.56 & 27.65 & 25.57 & 15.96 & 21.85 & 15.61 & 14.70 & 21.22 \\
\hline Authigenic & - & - & - & - & - & - & - & - & - & - \\
\hline Residual & - & - & - & - & - & - & - & - & - & - \\
\hline
\end{tabular}

\begin{tabular}{|c|c|c|c|c|c|c|c|}
\hline Sample & KASH-24 & KASH-26 & KASH-28 & KASH-20 & KASH-22 & KASH-30 & KASH-32 \\
\hline & \multicolumn{7}{|c|}{ mélange unit (accretionary wedge complex) } \\
\hline & \multicolumn{3}{|c|}{ matrix } & \multicolumn{4}{|c|}{ "exotic" blocks } \\
\hline Detrital & 81.89 & 83.62 & 82.13 & 75.64 & 61.82 & 69.02 & 68.35 \\
\hline Hydrothermal & - & - & - & - & - & - & - \\
\hline Biogenic & 18.11 & 16.38 & 17.87 & 24.27 & 38.17 & 29.35 & 31.44 \\
\hline Authigenic & - & - & - & 0.09 & - & - & - \\
\hline Residual & - & - & - & - & 0.01 & 1.63 & 0.21 \\
\hline
\end{tabular}

Note: The calculations were done using NORMSED program [Brandon, unpublished]

\section{Sandstones: Review of the Data Available and Their Interpretation}

The major-element composition of sandstones from the flysch and sedimentary mélange units on Karaginski Island was investigated by [Chamov, 1996] who concluded that the sandstones are derived from an ensialic arc. Analysis of the published data indicate that the sandstones show the dual geochemical affinity. On the one hand, they have high contents of $\mathrm{Fe}_{2} \mathrm{O}_{3 \text { tot }}+\mathrm{MgO}(7.62-10.81$ wt.\%) typical of sediments of oceanic island arcs and forearc basins. On the other hand, they are comparable with sandstones of differentiated continental arcs by $\mathrm{TiO}_{2}(0.55-0.91$ wt.\%) contents, and $\mathrm{Al}_{2} \mathrm{O}_{3} / \mathrm{SiO}_{2}(0.15-0.20), \mathrm{K}_{2} \mathrm{O} / \mathrm{Na}_{2} \mathrm{O}(0.33-1.25)$ and $\mathrm{Al}_{2} \mathrm{O}_{3} /\left(\mathrm{CaO}+\mathrm{Na}_{2} \mathrm{O}\right)(1.77-2.85)$ ratios. 

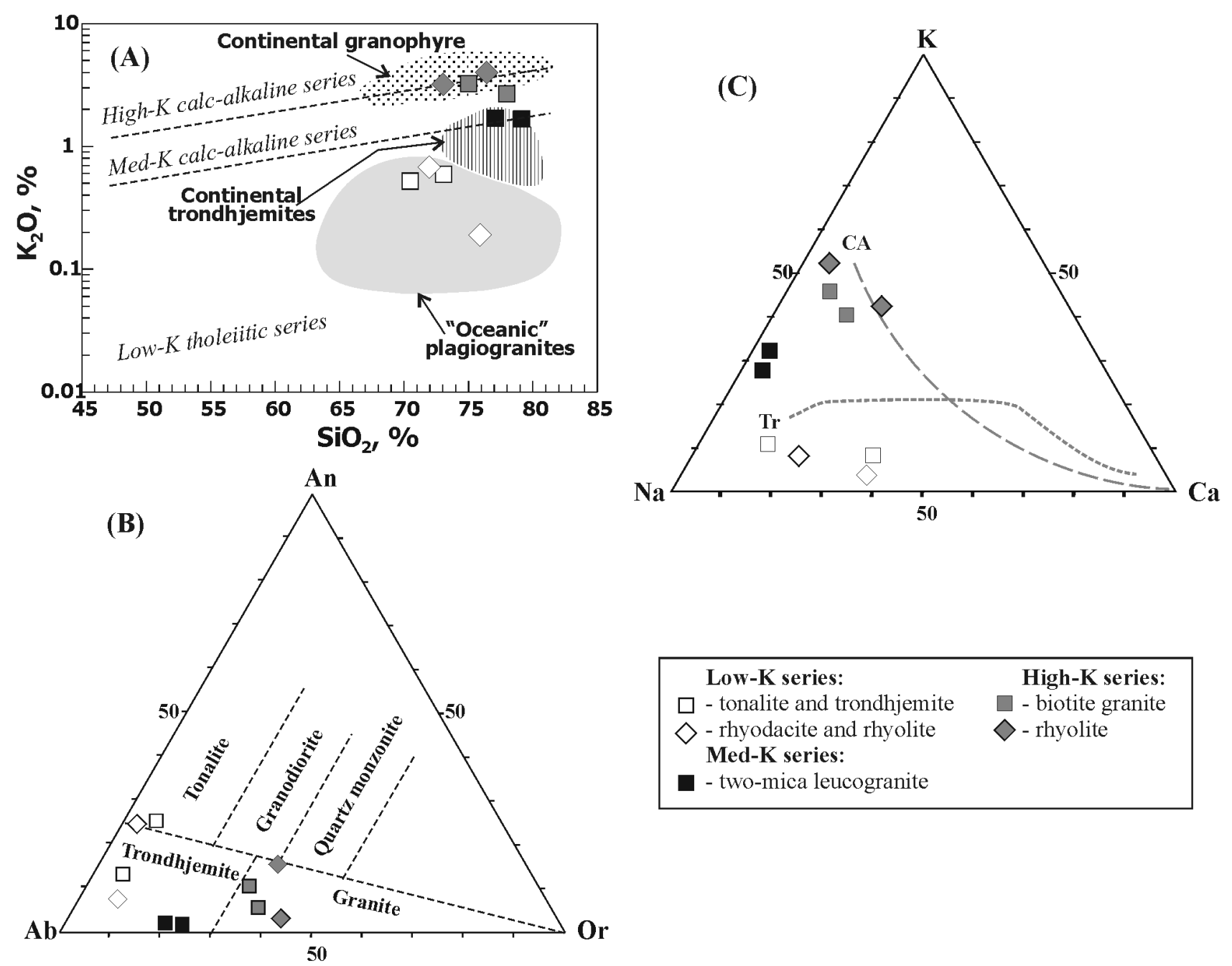

Figure 6. Plots $\mathrm{SiO}_{2}$ vs. $\mathrm{K}_{2} \mathrm{O}(\mathrm{a})$, Or- $\mathrm{An}-\mathrm{Ab}(\mathrm{b})$ and $\mathrm{Ca}-\mathrm{K}-\mathrm{Na}$ (c) demonstrating affinity of the studied felsic clasts to the low-K, med-K tonalitic-trondhjemitic and high-K calc-alkaline series. In Figure 5A, the fields of "oceanic" plagiogranites, continental trondhjemites and granophyres are after Coleman and Peterman [1975], and the discrimination lines of the low-K, med-K and high-K calc-alkaline series are after Peccerillo and Taylor [1976]. In Figure 4b, the classification boundaries are after O'Conner [1965]. In the Figure 4c, the trends of calc-alkaline (CA) and tonalite-trondhjemite $(\mathrm{Tr})$ series are after Baker and Arth [1976].

The chemical data available on the sandstones are well consistent with those obtained by us on the shales; and this is allow the suggestion that the sandstones and shales are genetically related to each other and have the common source of the detrital component. The latter is consistent with the observations made on the two-component diagrams, where different major elements are plotted against $\mathrm{SiO}_{2}$ concentrations and the sandstones and shales form single trends (Figure 4). The petrographic studies of sandstones from the flysch and mélange units done by Shapiro et al. [2000] showed that the sandstones are dominated in the debris of intrusive and extrusive felsic rocks and their minerals; and the latter are petrographically similar to the felsic cobbles embedded into the sediments in northeastern Karaginski Island. So, it can be concluded that the felsic cobbles carry out an important information on the provenance of the studied sediments.

\section{Felsic Cobbles: Geochemical Data and Their Interpretation}

The previous studies of sandstones indicate a provenance of felsic volcanic and plutonic rock detritus [Shapiro et al., 2000]. Several typical felsic rock cobbles from flysch and mélange were analyzed to evaluate their geochemical characteristics and to restrict a number of possible provenance sources of Karaginski Island sediments. The studied felsic cobbles chemically belong to the (a) low-K tonalite- 
trondhjemite, (b) med-K tonalite-trondhjemite, and (c) high-K calc-alkaline series (Figure 6).

\section{[a] Felsic Rocks of Low-K Tonalite-Trondhjemite Series}

The low-K felsic rocks are represented by rhyodacite, rhyolite, tonalite and trondhjemite that are petrographically similar but with variable geochemistry. The rhyolites and rhyodacites contain phenocrysts of sericitized plagioclase in a matrix of quartz, chlorite and subordinate amount of magnetite. The trondhjemite and tonalite are composed of plagioclase, quartz, biotite (replaced by chlorite), and subordinate alkali feldspar. Accessory phases include magnetite, apatite, and zircon.

The common feature of the low-K calc-alkaline felsic rocks is their significant depletion in $\mathrm{K}_{2} \mathrm{O}$ and as a result very low $\mathrm{K}_{2} \mathrm{O} / \mathrm{Na}_{2} \mathrm{O}$ values (0.06-0.13). In this regard, these rocks are similar to $\mathrm{M}$ - and I-type granitoids thought to originate from partial melting of primary magmatic protoliths or fractional crystallization of mantle-derived basaltic magma [Castro et al., 1991; Chappell and White, 1974; White and Chappell, 1977]. The high K/Rb (657-755) values at low $\mathrm{Rb} / \mathrm{Sr}(0.02-0.04)$ ratios indicate their affinity to M-type granitoids [Rozen, 2001]. But unlike those of M-type, the low-K calc-alkaline felsic cobbles are strongly peraluminous $\left(\mathrm{ASI}=\mathrm{Al}_{2} \mathrm{O}_{3} /\left(\mathrm{CaO}+\mathrm{Na}_{2} \mathrm{O}+\mathrm{K}_{2} \mathrm{O}\right)=1.18-1.38\right.$, molar $\left.\%\right)$ rocks with modal corundum, and, in this regard, they resemble S-type granitoids from crustal anatexis [Chappell and White, 1974; White and Chappell, 1977].

Despite a number of common features, the low-K calcalkaline felsic rock cobbles can be divided into two groups that differ in some major and trace element distribution. One group [group A1] includes trondhjemites low in $\mathrm{TiO}_{2}$ (0.19\%), $\mathrm{MgO}(0.50 \%)$ and $\mathrm{Fe}_{2} \mathrm{O}_{3}(1.62 \%)$, another one [group A2] is represented by the rhyolite, rhyodacite, and tonalite relatively rich in $\mathrm{TiO}_{2}(0.55-0.65 \%), \mathrm{MgO}(1.15-$ $2.36 \%)$ and $\mathrm{Fe}_{2} \mathrm{O}_{3}(3.78-4.41 \%)$. The trondhjemite [A1] LREE enrichment $\left((\mathrm{La} / \mathrm{Yb})_{\mathrm{N}}=17.69\right)$ coupled with the positive $\mathrm{Eu}\left(\mathrm{Eu} / \mathrm{Eu}^{*}=1.51\right)$ and no $\mathrm{Sr}\left(\mathrm{Sr} / \mathrm{Sr}^{*}=1.01\right)$ anomalies is consistent with crystallization from a primary melt produced by partial melting of an amphibolite or eclogite (Figure 7a-d) [Barker et al., 1976]. The REE and LILE/HFSE distribution indicates that the trondhjemite formation resulted from partial melting of a subducting slab material at high pressure. The high $\mathrm{Ba} / \mathrm{La}$ at high $\mathrm{La} / \mathrm{Yb}$ ratios (Figure 8a) suggest a composite source represented by a mixture of basalts and sediments.

The rhyolite and rhyodacite [A2] show features typical of products of a residue melt crystallization. Their moderate negative $\mathrm{Eu}\left(\mathrm{Eu} / \mathrm{Eu}^{*}=0.81-0.83\right)$ anomalies together with the fairly low $\mathrm{Sr}$ concentrations $\left(\mathrm{Sr} / \mathrm{Sr}^{*}=0.08-0.25\right)$ reflect prior plagioclase fractionation. The compositional variations of the rhyolite and rhyodacite can be related to fractional crystallization. The rhyolite in comparison with the rhyodacite is relatively abundant in $\mathrm{MgO}, \mathrm{Fe}_{2} \mathrm{O}_{3}$ at the lower $\mathrm{FeO}^{*} / \mathrm{MgO}$ value, alkali contents, less fractionated LREE and higher $\mathrm{Sr} / \mathrm{Sr}^{*}$ ratio. The rhyodacite and tonalite are very similar in composition and are likely comagmatic.
The rhyolite shows slight LREE fractionation $\left((\mathrm{La} / \mathrm{Yb})_{\mathrm{N}}\right.$ $\left.=1.41,(\mathrm{La} / \mathrm{Sm})_{\mathrm{N}}=1.44\right)$ and the rhyodacite shows moderate enrichment in LREE $\left((\mathrm{La} / \mathrm{Yb})_{\mathrm{N}}=5.99,(\mathrm{La} / \mathrm{Sm})_{\mathrm{N}}=\right.$ 3.33) coupled with their nearly equal abundance in HREE $\left((\mathrm{Gd} / \mathrm{Yb})_{\mathrm{N}}=0.93\right.$ and 1.15$)$ and $\mathrm{Y}(13.65$ and $14.40 \mathrm{ppm}$ in rhyolite and rhyodacite, respectively) and pronounced negative $\mathrm{Nb}\left(\mathrm{Nb} / \mathrm{Nb}^{*}=0.24\right)$ and $\mathrm{Ta}\left(\mathrm{Ta} / \mathrm{Ta}^{*}=0.03\right)$ anomalies indicate that it is a primary melt originated by a partial melting of depleted mantle peridotite in a subduction zone. Partial melting of mantle peridotite results in a wide range of compositions varying from basaltic to andesitic [Baker et al., 1995; Falloon et al., 1997; Robinson et al., 1998 and etc.]. Mantle-derived intermediate magmas are commonly produced by a low-degree partial melting [Baker et al., 1995; Robinson et al., 1998 and etc.], and their peculiar geochemical feature is originally fractionated LREE distribution. So, it is unlikely that the low LREE fractionated rhyolite represents the residue melt produced from a mantle-derived intermediate magma after quartz, plagioclase and biotite accumulation.

The rhyolite high $\mathrm{Ba} / \mathrm{La}$ ratios at low $\mathrm{La} / \mathrm{Yb}$ (Figure 8a) and the low $\mathrm{Ti} / \mathrm{Hf}$ at increased La/Ta (Figure $8 \mathrm{~b}$ ) allow us to suggest that the source was metasomatically reworked by slab-derived hydrous fluids and/or melts, and that the partial melting took place under hydrous conditions [Lin et al., 1989; Yagodzinski et al., 1995]. The partial melting of such mantle under hydrous conditions should produced low-K and high-Al andesitic melt. The crystallization of the studied rhyolite, rhyodacite and tonalite from the low-K and high$\mathrm{Al}$ andesitic melt is very attractive by a number of reasons. It might explain (a) their high ASI numbers; (b) their lower HREE contents and absence of the pronounced negative Eu anomalies; and (c) allow us to understand the simultaneous appearance of mantle- and slab-derived felsic rocks of the tonalite-trondhjemite series. Thus, the intrusive and effusive rocks of the low-K calc-alkaline series directly and indirectly relate to each other. The compositional variations of the rhyolite, rhyodacite, trondhjemite and tonalite indicate formation in a subduction setting.

\section{[b] Felsic Rocks of Med-K Tonalite-Trondhjemite Series}

These rocks are represented by two-mica leucogranites of very similar petrographical and geochemical composition. They are composed of zoned plagioclase partially substitute by sericite and potassium feldspar, which are present in nearly equal amount, quartz, muscovite, and biotite (replaced by alkaline chlorite and magnetite). Accessory minerals include topaz, apatite, zircon and magnetite.

The two-mica leucogranites $\left(\mathrm{SiO}_{2}=77.08-79.12\right.$ wt.\%) show dual geochemical affinity. As to $\mathrm{K}_{2} \mathrm{O}, \mathrm{Rb}$ and $\mathrm{Sr}$ abundances and their low $\mathrm{K}_{2} \mathrm{O} / \mathrm{Na}_{2} \mathrm{O}(0.36-0.45)$ and $\mathrm{Rb} / \mathrm{Sr}$ (0.31-0.44) ratios, they are similar to continental trondhjemites (Figure 6a) and can be classed as the granitoids of I-type. On the other hand, the presence of muscovite and biotite, and high aluminum saturation index $($ ASI $=1.25-1.37)$ indicate their similarity to crustally 


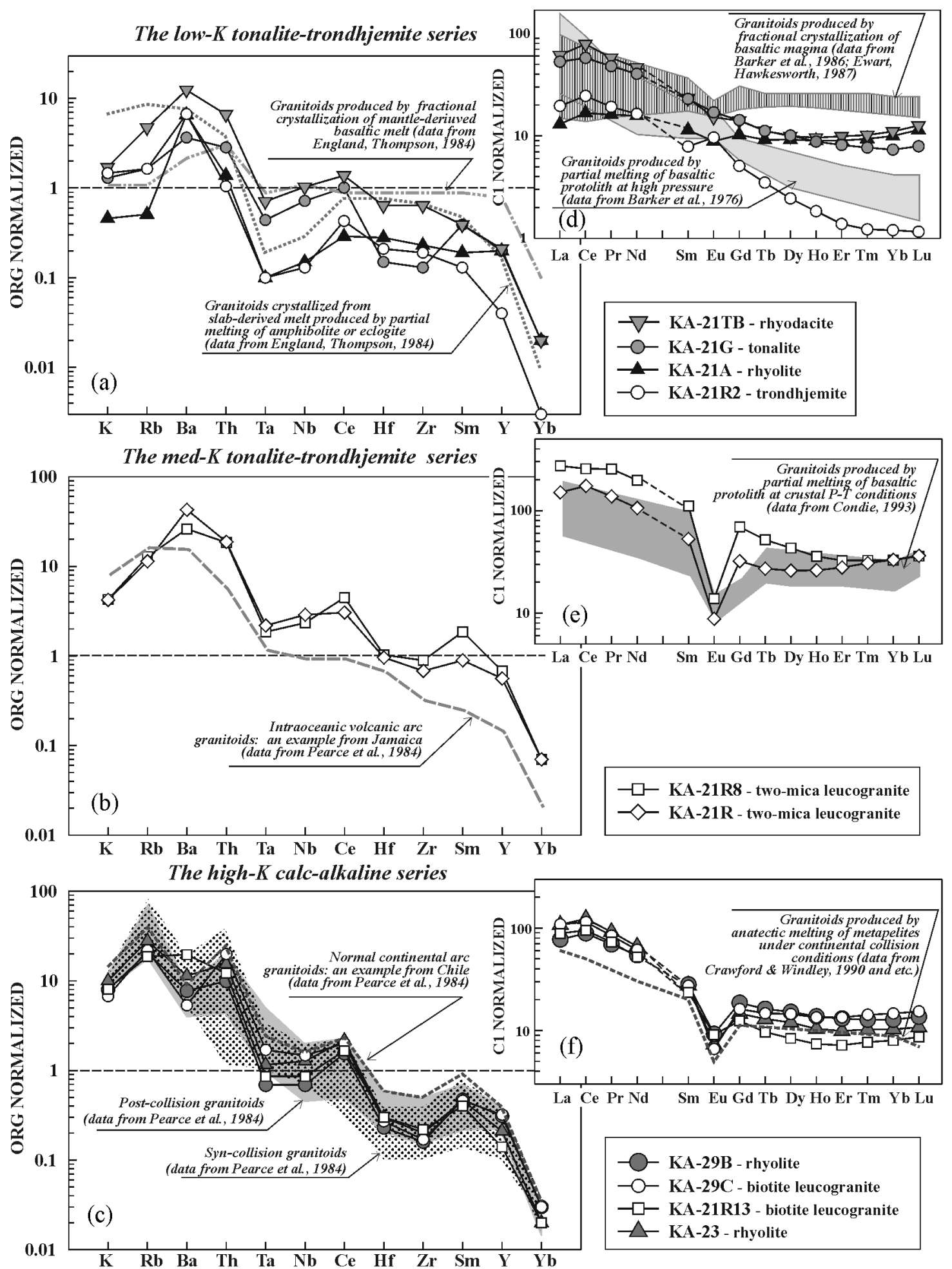

Figure 7. Normalized multi-element $(\mathrm{a}-\mathrm{c})$ and REE $(\mathrm{d}-\mathrm{f})$ spectra for the felsic clasts in the sediments of northeastern Karaginski Island demonstrating the similarity of the studied felsic rocks to granitoids produced by different processes and those generated in different tectonic settings. Normalizing values are after Pearce et al. [1984] for ORG (oceanic ridge granite) and Anders and Grevesse [1989] for chondrite. 

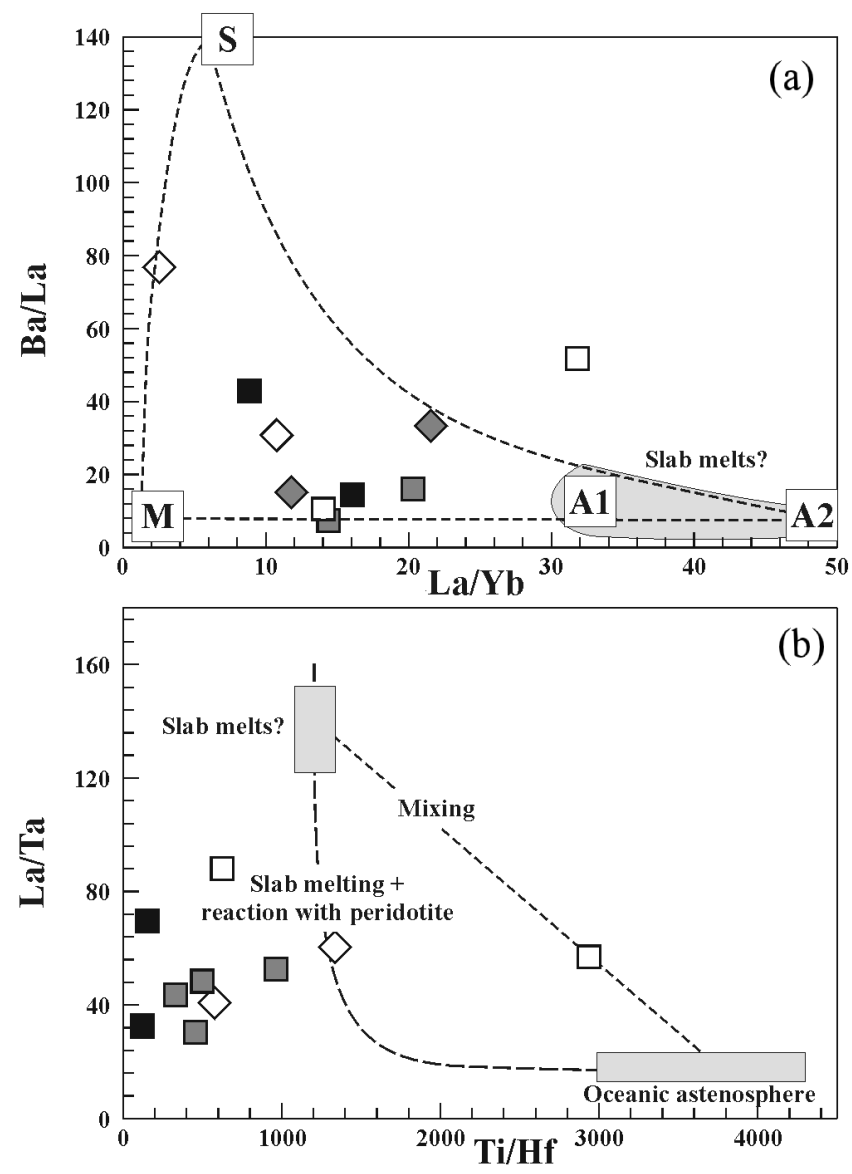

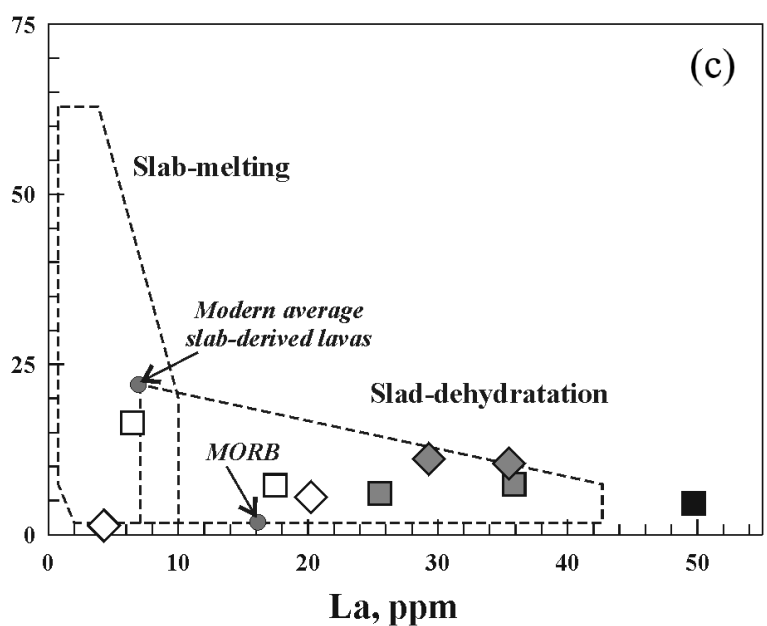

\begin{tabular}{|lc|}
\hline Low-K series: & High-K series: \\
$\square$ - tonalite and trondhjemite & - biotite granite \\
$\diamond$ - rhyodacite and rhyolite & $\diamond$ - rhyolite \\
Med-K series: & \\
- two-mica leucogranite & \\
\hline
\end{tabular}

Figure 8. La/Yb vs. Ba/La (a), Ti/Hf vs. La/Ta (b) (both after [Yagodzinski et al., 1995]) and La vs. $(\mathrm{La} / \mathrm{Yb})_{\mathrm{N}}(\mathrm{c})$ (after $[$ Martin, 1986, 1993]) plots demonstrating influence of different source components on the compositional variations of the studied felsic clasts.

derived S-type granitoids. Moreover, in the discrimination diagrams (Figure 9), the two-mica leucogranites either lie along the lines separating fields of the volcanic-arc and within-plate granitoids or fall in the field of the within-plate granitoids. The main question arises now as to whether the formation of the two-mica leucogranites relates to subduction or lithospheric extension processes.

The direct comparison of the multi-element spectra with those typical of granitoids from various geodynamic setting do not allow us to resolve this problem unambiguously. On the one hand, the two-mica leucogranite LILE-HFSE patterns show the principal similarity to those typical of intraoceanic volcanic arc granitoids (Figure $7 \mathrm{~b}$ ). In particular, they are characterized by the enhanced enrichment in LILE such as $\mathrm{K}_{2} \mathrm{O}, \mathrm{Rb}, \mathrm{Ba}$ and $\mathrm{Th}\left(\mathrm{K}_{2} \mathrm{O}<\mathrm{Rb}<\mathrm{Ba}\right.$ and $\left.\mathrm{Ba}>\mathrm{Th}\right)$ relative to HFSE and presence of the pronounced negative $\mathrm{Nb}\left(\mathrm{Nb} / \mathrm{Nb}^{*}=0.19-0.26\right)$ and $\mathrm{Ta}\left(\mathrm{Ta} / \mathrm{Ta}^{*}=0.09\right)$ anomalies. On the other hand, the levels of HFSE such as Nb, Ta, $\mathrm{Zr}$, Hf and Y are much higher than in those typical of the intraoceanic volcanic arc granitoids.

The analysis of the REE distribution in the two-mica leucogranites allow us to suggest that the two-mica leucogranites originated by partial melting of basaltic source under crustal PT conditions (Figure 7e). This conclusion is consis- tent with high REE levels, moderate fractionation of LREE $\left((\mathrm{La} / \mathrm{Yb})_{\mathrm{N}}=4.92-8.95\right)$ at flat HREE patterns $\left((\mathrm{Gd} / \mathrm{Yb})_{\mathrm{N}}\right.$ $=0.88-1.91)$ and strong negative $\mathrm{Eu}\left(\mathrm{Eu} / \mathrm{Eu}^{*}=0.16-0.21\right)$ anomalies. Taken into account the REE and other trace element behavior, it can be concluded that the two-mica leucogranites inherited the geochemical affinity of the melted basaltic source that was originally created by subduction zone processes.

If the proposed petrological model for the formation of the two-mica leucogranites is correct, then we should expect that the melted basalt compositionally corresponded to high-Al calc-alkaline basalt slightly or moderately enriched in LREE. Thus, the two-mica leucogranite most probably originated by partial melting of subduction zone mantlederived basaltic source at crustal conditions.

\section{[c] Felsic Rocks of High-K Calc-Alkaline Series}

These rocks are represented by biotite leucogranites, rhyolites and their tuffs. Petrographically, the granites are composed of euhedral crystals of zoned plagioclase partially replaced by saussurite, microcline, biotite commonly replaced by chlorite and magnetite and interstitial quartz. Accessory 

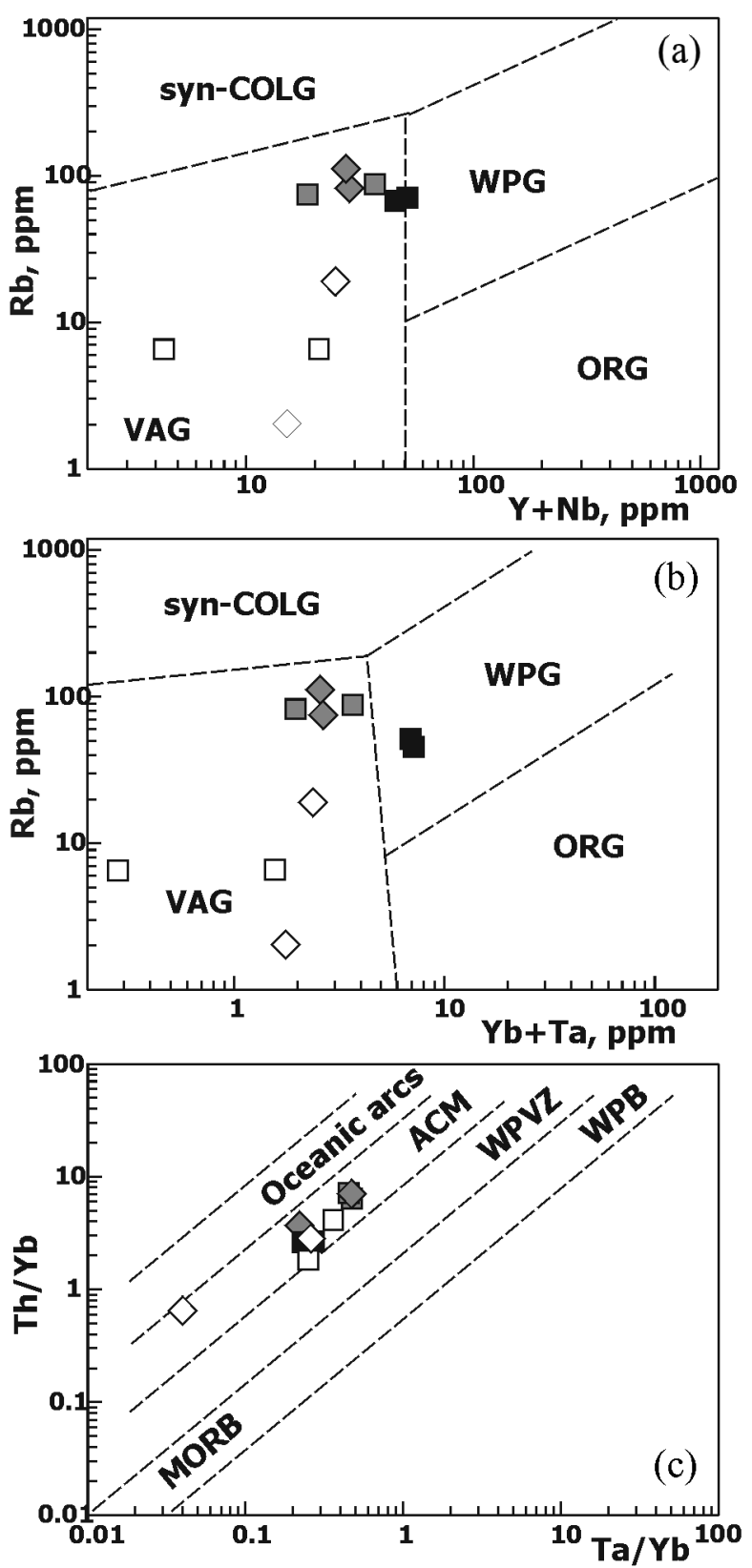

\begin{tabular}{lc}
\hline Low-K series: & High-K series: \\
$\square$ - tonalite and trondhjemite & $\square$ - biotite granite \\
$\diamond$ - rhyodacite and rhyolite & $\diamond$ - rhyolite \\
Med-K series: & \\
& - two-mica leucogranite
\end{tabular}

Figure 9. Discriminant diagrams $\mathrm{Rb}$ vs. $(\mathrm{Y}+\mathrm{Nb})(\mathrm{A}), \mathrm{Rb}$ vs. (Yb+Ta) (B) (both after [Pearce et al., 1984]) allow the recognition of syn-collisional (syn-COLG), within plate (WPG), oceanic ridge (ORG) and volcanic arc (VAG) granitoids and $\mathrm{Ta} / \mathrm{Yb}$ vs. Th/Yb (C) (after [Gorton and Schandl, 2000]) enable the recognition of volcanics formed in different tectonic settings: active continental margin (ACM), withinplate volcanic zones (WPVZ), within-plate basalts (WPB), and mid-ocean ridge basalts (MORB). minerals include apatite, zircon, sillimanite and oxidized magnetite. The rhyolites and their tuffs contain phenocrysts of altered plagioclase, potassium feldspar and quartz in the matrix of albite, chlorite and quartz.

The rhyolites and biotite leucogranites are peraluminous $(\mathrm{ASI}=1.18-1.31)$ rocks almost equally abundant in $\mathrm{Na}_{2} \mathrm{O}$ and $\mathrm{K}_{2} \mathrm{O} \quad\left(\mathrm{Na}_{2} \mathrm{O} / \mathrm{K}_{2} \mathrm{O}=0.81-0.90\right.$ in granites and 1.03-1.11 in rhyolites). In this respect, they are similar to S-type granites. These rocks are characterized by relatively high $\mathrm{K} / \mathrm{Rb}(255-359)$ and variable $\mathrm{Rb} / \mathrm{Sr}(0.49-2.01)$ ratios. As to the trace element abundance and distribution, the high-K calc-alkaline felsic rock cobbles show the affinity to both normal continental arc and continent-arc collision (synorogenic) granitoids (Figure 7c). They are characterized by significant enrichment in LILE relative to HFSE, presence of $\mathrm{K}_{2} \mathrm{O}, \mathrm{Rb}, \mathrm{Ba}$ and $\mathrm{Th}$ extremes and pronounced negative $\mathrm{Nb}\left(\mathrm{Nb} / \mathrm{Nb}^{*}=0.17-0.22\right)$ and $\mathrm{Ta}\left(\mathrm{Ta} / \mathrm{Ta}^{*}=0.07-0.18\right)$ anomalies. The differentiated $\operatorname{LREE}\left((\mathrm{La} / \mathrm{Yb})_{\mathrm{N}}=6.56^{-}\right.$ $1.99)$ at flat $\operatorname{HREE}\left((\mathrm{Gd} / \mathrm{Yb})_{\mathrm{N}}=1.00-1.40\right)$ and negative $\mathrm{Eu}\left(\mathrm{Eu} / \mathrm{Eu}^{*}=0.31-0.53\right)$ anomalies is consistent with anatectic melting of metapelites (Figure $7 \mathrm{f}$ ).

\section{Results of FT Dating of Zircons From Felsic Cobbles}

Several granitic and rhyolitic cobbles belonging to different geochemical types were dated using fission-track method to elucidate the provenance of the felsic clasts in the sediments. The felsic rock cobble of all types have a range of zircon FT grain ages that indicate cooling in the source in the Late Cretaceous to Early Eocene (Tables 3 and 7). Tonalites and trondhjemites (low-K) cooled at $53.8(-3.5 /+3.8) \mathrm{Ma}$ and $78.5(-6.7 /+7.6)$ Ma, respectively. The rhyolites (med-K) cooled at $70.1(-3.7 /+3.9)$ Ma. The biotite leucogranites (high-K) cooled between $59.1 \mathrm{Ma}(-3.3 /+3.9)$ and $58.6(-3.2 /+3.3) \mathrm{Ma}$ (two clasts). The Late Cretaceous cooling ages (70-78 Ma) probably reflect original magmatic activity whereas the Early Eocene cooling ages may indicate timing of exhumation.

The zircon FT grain ages for the felsic cobbles are older than the depositional age of sandstones (c. 45 to $26 \mathrm{Ma}$ ) that dominate rocks in the sedimentary mélange [Garver et al., 2001]. The implications of these observations are that several sources of different ages contributed to these sediments. This is also consistent with the paleontological age determinations that indicate that some shales contain nannoplankton species indicative of the upper Cretaceous and Paleogene intervals [Shapiro et al., 2004]. We suggested that a provenance of the youngest component can be reconstructed based on the analysis of geochemical features of sediments and a provenance of the older component based on the analysis of petrochemical and geochemical features of felsic cobbles. 
Table 8. Indicative ratios in shales of the accretionary complex in Karaginski Island in comparison with those in volcanics of the Western-Kamchatka - Koryak volcanic belt, PAAS and estimated bulk continental crust

\begin{tabular}{|c|c|c|c|c|c|}
\hline Deposits & $\mathrm{La} / \mathrm{Sc}$ & $\mathrm{Th} / \mathrm{Sc}$ & $\mathrm{Cr} / \mathrm{Ni}$ & $\mathrm{La} / \mathrm{Yb}$ & Source of data \\
\hline \multicolumn{6}{|c|}{ Sediments of the accretionary complex in Karaginski Island } \\
\hline $\begin{array}{l}\text { Shales of the transitional } \\
\text { volcanic-sedimentary unit }(\mathrm{N}=8)\end{array}$ & $\begin{array}{c}1.00-1.84 \\
(1.18)\end{array}$ & $\begin{array}{l}0.38-0.48 \\
(0.42)\end{array}$ & $\begin{array}{l}1.94-2.60 \\
(2.38)\end{array}$ & $\begin{array}{l}3.79-5.58 \\
(4.88)\end{array}$ & this paper \\
\hline $\begin{array}{l}\text { Shales of the flysch unit } \\
(\mathrm{N}=10)\end{array}$ & $\begin{array}{l}1.06-1.40 \\
(1.37)\end{array}$ & $\begin{array}{l}0.30-0.38 \\
(0.35)\end{array}$ & $\begin{array}{l}0.95-2.82 \\
\quad(2.29)\end{array}$ & $\begin{array}{l}4.14-5.48 \\
\quad(4.64)\end{array}$ & this paper \\
\hline $\begin{array}{l}\text { Shales of the sedimentary } \\
\text { mélange unit }(\mathrm{N}=5)\end{array}$ & $\begin{array}{c}1.08-1.49 \\
(1.29)\end{array}$ & $\begin{array}{l}0.31-0.45 \\
\quad(0.38)\end{array}$ & $\begin{array}{l}0.50-2.94 \\
\quad(2.45)\end{array}$ & $\begin{array}{l}2.58-9.97 \\
(4.35)\end{array}$ & this paper \\
\hline \multicolumn{6}{|c|}{ Volcanics of the Western Kamchatka - Koryak volcanic belt } \\
\hline $\begin{array}{l}\text { Mantle-derived felsic volcanics } \\
(\mathrm{N}=16)\end{array}$ & $\begin{array}{l}0.60-1.58 \\
\quad(0.71)\end{array}$ & $\begin{array}{l}0.13-0.51 \\
\quad(0.18)\end{array}$ & $\begin{array}{l}1.43-3.86 \\
(2.45)\end{array}$ & $\begin{array}{l}2.58-9.97 \\
(4.35)\end{array}$ & [Shantser and Fedorov, 1997] \\
\hline $\begin{array}{l}\text { Crustally-derived felsic volcanics } \\
(\mathrm{N}=10)\end{array}$ & $\begin{array}{l}0.25-1.80 \\
(1.01)\end{array}$ & $\begin{array}{l}0.05-0.58 \\
\quad(0.28)\end{array}$ & $\begin{array}{l}1.16-4.76 \\
(2.20)\end{array}$ & $\begin{array}{l}2.89-6.99 \\
(5.40)\end{array}$ & [Shantser and Fedorov, 1997] \\
\hline PAAS & 2.38 & 0.91 & 2.08 & 6.99 & [Taylor and McLennan, 1985] \\
\hline Estimated bulk continental crust & 0.53 & 0.12 & - & 3.75 & [Hofmann, 1988] \\
\hline
\end{tabular}

Note: average concentrations are shown in brackets; $\mathrm{N}$ is the number of analysis.

\section{Discussion}

The zircon FT grain ages for the felsic cobbles are older than the depositional age of sandstones (c. 46 to $20 \mathrm{Ma}$ ) [Garver et al., 2001; Shapiro et al., 2004]. The flysch and mélange have paleontological ages that range from the middle Eocene to early Miocene [Chekhovich et al., 1990; Shapiro et al., 2004] which are similar to the young peak ages in the sandstones. The implications of these observations are that: (a) several sources contributed to these sediments; (b) the youngest detrital component most probably derived from a volcanic source being active through the middle Eocene to early Miocene; and (c) the felsic rock cobbles represent the essentially random selection of the older material component probably derived from pre-collisional structures of the paleoAsian continental margin. Note that we were able to collect a few paleocurrent measurements (only 8) from flutes and grooves on the base of sandstone beds. These flutes show transport to about $120^{\circ}$ which is nearly perpendicular to the trend of the modern margin of this part of Kamchatka $\left(\sim 40^{\circ}\right)$. We do not have paleocurrent measurements from the pebbly mudstone units, but we do know that the FT peak ages in the sandstones and the cobbles are similar suggesting a common source [Garver and Soloviev, 2001, unpublished data].

\section{Possible Provenance Source of the Youngest Detrital Component}

The timing of sediment deposition (c. 46-20 Ma) coincides with timing of magmatism occurring in the Western Kamchatka - Koryak volcanic belt formed by west-directed subduction beneath the convergent margin of paleo-Asian continent [Filatova, 1988]. The volcanics and related volcanic-sedimentary deposits of this belt are dated at 46.5 $\pm 0.3-37.4 \pm 0.6 \mathrm{Ma}$ [Bagdasaryan et al., 1996; Soloviev et al., 2002].

The geochemical features of volcanics of the Western Kamchatka - Koryak volcanic belt also indicate that they may have made a significant contribution to the accretionary wedge now exposed on Karaginski Island (Figure 5b). These volcanics are dominated by the calc-alkaline intermediate and felsic volcanics [Shantser and Fedorov, 1997] of two genetically different types. Felsic rocks resulted from the fractional crystallization of melts produced by partial melting of mantle peridotite and anatectic melting of sediments. Both mantle- and crustally-derived volcanics are characterized by the low incompatible/compatible element ratios (Table 8). The shales on Karaginski have similar geochemical features. The volcanics are also low in the transitional metals such as $\mathrm{Cr}, \mathrm{Co}, \mathrm{Ni}$ and have the low $\mathrm{Cr} / \mathrm{Ni}$ ratios comparable to those in the shales of the accretionary wedge (Table 8). Thus, we conclude that the youngest detrital component in the accretionary wedge sediments probably represents syncontemporaneous volcanic material most probably derived from volcanics of the Western Kamchatka - Koryak volcanic belt.

\section{Possible Provenance Sources of One of the Older Detrital Components}

The simple pattern of cooling ages from cobbles of different geochemical types, combined with fact that the rounded cobbles occur together in thin, discreet beds undoubtedly 

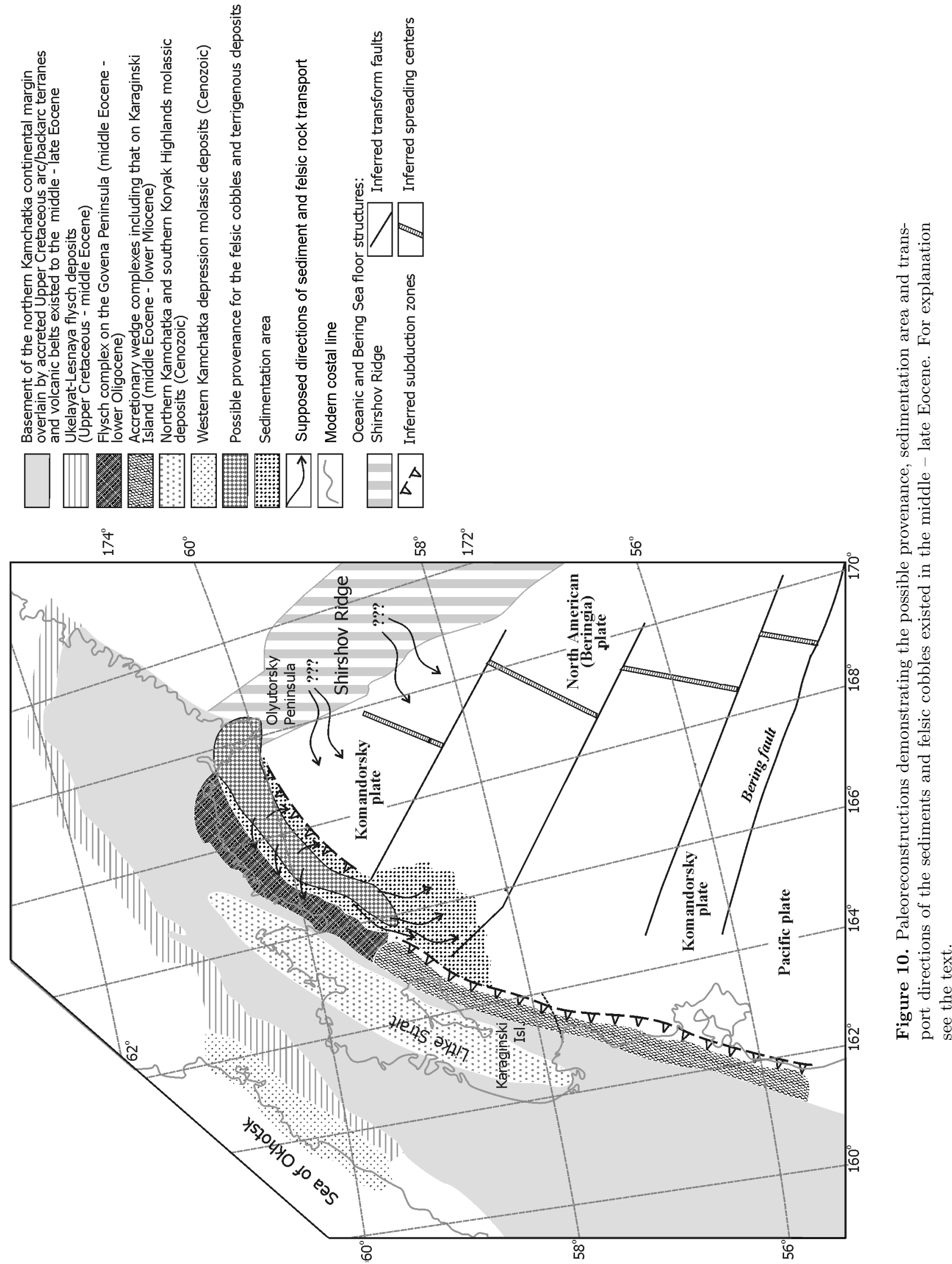
indicates derivation from a single heterogeneous source. Because the felsic cobbles originated from melts produced by partial melting of (a) slab and depleted mantle peridotites reworked by fluids from the subducting slab, and (b) anatectic melting of basic volcanics and sediments, this source was formed either by: [a] subduction in the Late Cretaceous and collision/exhumation in the Early Paleogene; or by [b] continuous subduction.

\section{Constraints for a Provenance Source of the Older Detrital Component}

The principal aspect of any such reconstruction is the position of the Litke Basin (Figure 1), which largely rests below the modern Litke Strait, but its strata are exposed along the margin of Karaginski Island. The stratigraphy and sediment composition of these strata are well known and it seems that the basin persisted as a simple trough-like feature from the Eocene to the present as there are no unconformities or significant breaks in deposition. Two issues arise from this observation. First, these strata do not contain felsic cobbles as are present in the accretionary prism just outboard. Second, this trough-like basin would have acted as a sediment trap and therefore would have significantly impeded sediment transport out of the basin and into the accretionary prism. As such, any paleogeographic reconstruction of sediment dispersal from the volcanic arc and surrounding basement rock has to consider the Litke Basin as an obstacle to sediment transport from exposed mainland to the accretionary prism. Thus, reasonable paleogeodynamic reconstructions for the onset of deposition of rocks now in the accretionary wedge (middle Eocene to early Miocene) would include geological structures located to the east of the Litke Strait. The possible candidates are as follows: (a) the Govena-Karaginski, (b) Olyutorsky Ridge, and (c) Olyutorsky Peninsula subterranes.

The derivation of the felsic material from the GovenaKaraginski and Olyutorsky Ridge subterranes is unlikely. The upper Cretaceous igneous rocks of the GovenaKaraginski subterrane are dominated by ultramafics, tholeiitic gabbro and amphibolites and tholeiitic and calc-alkaline subvolcanic and volcanic rocks of ophiolite assemblages, med-K and high-K calc-alkaline basaltic and basaltictrachybasaltic series [Chekhovich et al., 1990; KravchenkoBerezhnoy and Nazimova, 1991]. The significant amount of ultramafics among the Govena-Karaginski subterrane assemblage allows us to exclude this structure from possible sources of the Karaginski Island sediments as the geochemical features of the latter indicate negligible contribution of ultramafics. The upper Cretaceous igneous rocks of the Olyutorsky Ridge subterrane are dominated by calcalkaline basalts and basaltic andesites [Sukhov, 1983] and pyroxenite-gabbro-diorite arc-root complexes [Batanova and Astrakhantsev, 1992, 1994; Kepezhinskas et al., 1993; Ledneva et al., 2000] that are petrographically and chemically different from igneous rock assemblages found in cobbles. However, the terrane also has small intrusions of diorites, granodiorites and granites but they are inferred to be the Paleocene-Eocene in age [Kepezhinskas and Savichev, 1991; Kepezhinskas et al., 1991].

Within the Olyutorsky accretionary-collision zone we have identified only one likely candidate source referred to as the Olyutorsky Peninsula subterrane at the Cape, that have the right age and similar major-element composition (geochemistry of these rocks is poorly studied) to the cobbles in the accretionary prism. The evolution of the Olyutorsky Peninsula subterrane being at tectonic contact with the structures of the Olyutorsky Ridge is poorly understood. The Olyutorsky Peninsula is composed of: (a) tectonically juxtaposed upper Senonian oceanic complexes [Bogdanov and Khain, 2000b; Bogdanov and Tilman, 1991; Bogdanov et al., 1987]; and (b) upper Cretaceous and Eocene felsic extrusive, subvolcanic and plutonic rocks. The data available on the extrusive felsic and subvolcanic rocks of the Olyutorsky Peninsula suggest that they belong to low-K and med-K tonalite - trondhjemite and high-K calc-alkaline series and petrochemically very similar to the corresponding types of granitoids from the felsic cobbles in the accretionary wedge sediments in Karaginski Island.

\section{Unresolved Problems and Geodynamic Speculations}

The Olyutorsky Peninsula subterrane located at the distance of about $350 \mathrm{~km}$ from Karaginski Island is too small to produce material enough for the formation of aureole about $300 \mathrm{~km}$ long and $20 \mathrm{~km}$ wide at the structural thickness of about $5 \mathrm{~km}$ (Figure 10). Therefore, underwater structures of the Komandosky basin should be considered. The structure of the Olyutorsky Peninsula morphologically continues the Shirshov Ridge that was previously considered as a potential source of the northeastern Karaginski sediments and felsic cobbles [Shapiro, 1984]. We speculate that there is another possibility as well. At the mainland shelf between the Olyutorsky Peninsula and Karaginski Island, there are several underwater highs, which form the discontinuous chain (Figure 1). These highs are covered by the water column of about 150 meters thick and separated from each other and from underwater southwestern continuation of the Govena Peninsula by depressions of 600-1000 meters deep. Morphological features of the highs indicate that they were eroded and flattened before the subsidence. We don't know what geological complexes compose these structures. But if the felsic material in northeastern Karaginski Island derived from them, we are able to propose the kinematic scheme explaining the formation of the accretionary wedge sediments, which does not contradict with the modern viewpoints on the Komandorsky basin structure and its framing.

This model is based on the assumptions as follows: (a) the structure and configuration of the considered part of the Eurasian mainland were similar to its present-day structure and configuration since the middle Eocene; and (b) the Shirshov Ridge was in its present position since the middle Eocene. The Komandorsky basin itself was the part of the Pacific plate until the middle Eocene and moved along with this plate to the north relative to Eurasia. The Shirshov Ridge and Komandorsky basin were presumably 
separated by transform fault. The change in the Pacific plate motion direction onto the northwestern one was resulted in establishment of the Bering fault and separation of the Komandorsky basin plate from the Pacific one. The later scenario of the Komandorsky basin development is characterized by initiation of numerous rifts of the northeastern strike in its southern part adjacent to the Shirshov Ridge. These rifts were separated from each other by transform faults of the northwestern strike and the northernmost one of them separated the highs in the south. Simultaneously the trench that continues the Kuril-Kamchatka one was established along the northwestern edge of the basin, the subduction began along this trench, and the accretionary wedge started to form.

The subduction most probably did not took place north of Karaginski Island. Nonetheless this segment where the highs are located experienced some extension and compression. The later probably resulted in uplift of the highs and its intensive erosion. The products of this erosion were transported not only in the northwestern and southeastern directions but also in the southern direction and were accumulated at the oceanic plate moved in the northwestward direction. The plate, therefore, carried these sediments toward the trench. This scenario is also enable us to explain occurrence of the oceanic basalt fragments in the accretionary wedge in the northeastern part of Karaginski Island.

\section{Conclusions}

1. The compositional variations of the studied shales in northeastern Karaginski Island indicate that (a) they were probably deposited in both forearc and oceanic settings, and (b) the detrital material dominated in sediments from these different tectonic settings was mainly derived from a differentiated continental or island arc. The compositional variations of the shales and sandstones composing the accretionary wedge in northeastern Karaginski Island are consistent with common genesis of these sediments. The previous studies [Shapiro, 1984; Shapiro et al., 2000] of sandstones showed that the latter are dominated by debris of felsic intrusive and extrusive rocks, and that these debris closely resemble felsic igneous rocks found as cobbles and boulders in the sediments of the flysch and mélange units.

2. The felsic rock cobble have a range of zircon FT grain ages that indicate cooling in the source in the Late Cretaceous to Early Eocene. The zircon FT grain ages for the felsic cobbles are older than the depositional age of sandstones (c. 46 to $20 \mathrm{Ma}$ ) [Garver et al., 2001; Shapiro et al., 2004] but the former coincide with P2 zircon ages of sandstones. The implications of these observations are that (a) several sources of different ages contributed to these sediments, and (b) the felsic cobbles provide the information on one of the older detrital components of the studied sediments.

3. The cobbles include three distinct types: low-K, med-K and high-K calc-alkaline igneous rocks. [A] The low$\mathrm{K}$ cobbles are tonalitic and trondhjemitic between M- and I-type granitoids and their effusive analogues (rhyodacite, rhyolite), which may be genetically related to each other and generated from low-K, high-Al andesitic melt produced by: (i) partial melting of mantle metasomatically reworked by slab-derived hydrous fluids and/or melts under hydrous conditions; or (ii) a subducting slab material at high pressure. [B] The med-K cobbles are two-mica leucogranites with a dual geochemical affinity, and are similar to both I-type and crustally derived S-type granitoids, and are probably formed by partial melting of subduction zone mantle-derived basaltic source at lower crustal PT conditions. [C] The high$\mathrm{K}$ cobbles are biotite leucogranites, rhyolites, and their tuffs, which are similar to S-type granitoids from anatectic melting of metapelites.

Combining the data on chemistry and ages of felsic cobbles, it can be concluded that one of the older detrital component in the sediments of northeastern Karaginski Island derived from the region formed by (a) subduction in the Late Cretaceous and followed by exhumation in the Paleocene; or by (b) continuous subduction in the Late Cretaceous Paleogene.

4. The closest petrochemical analogues of the cobbles occur only locally in the Olyutorsky Peninsula subterrane. Nonetheless, this structure is too small and too remote from Karaginski Island to supply enough material for the middle Eocene - lower Miocene sediments of the accretionary wedge. We suggested that the Olyutorsky Peninsula subterrane structure might be extended in the shelf where several highs exist.

5. The youngest detrital component (the middle Eocene - early Miocene) most probably represents volcanic material from the Western Kamchatka-Koryak volcanic belt that was active at the time of the accretionary wedge sediment deposition.

Acknowledgments. The research described in this paper was made possible in part by Award No RG1-2568-MO-03 of the U.S. Civilian Reseach \& Development Foundation for the Independent States of the Former Soviet Union (CRDF) to A. V. Soloviev and J. I. Garver and by Award No. EAR - OPP 99-11910 of the National Science Foundation of the USA to J. I. Garver and M. T. Brandon. The authors are grateful to N. A. Bogdanov, the director of the Institute of the Lithosphere of Marginal Seas RAS in Moscow, supported the collaborative effort between the authors and field trips since 1993.

\section{References}

Achmetiev, M. A., A. I. Dvoryankin, S. Milyokhin et al. (1989), Paleocene chrebta Raretkyn - Paleocene of the Raretkyn Ridge, Izv. AN SSSR, Ser. geology (in Russian), 3, 44-58.

Anders, E., and N. Grevesse (1989), Abundances of the elements: Meteoritic and Solar, Geochim. Cosmochim. Acta, 53, 197214.

Bagdasaryan, G. P., Yu. B. Gladenkov, A. E. Shantser (1994), Isotope dating of the Paleogene and Neogene volcanic sequences of Kamchatka, Stratigraphy and Geological Correlation (in Russian), 2, 87-91.

Baker, M. B., M. M. Hirshmann, M. S. Ghiorso, and E. M. Stolper (1995), Compositions of the near-solidus peridotite melts from experiments and thermodynamic calculations, Nature, 375, 308-311.

Baranov, B. V., N. I. Seliverstov, A. V. Murav'ev, and E. L. Muzurov (1991), The Komandorsky basin a product of spreading behind a transform plate boundary, Tectonophysics, 199, 237-269. 
Barker, F., and A. G. Arth (1976), Generation of trondhjemitictonalitic liquids and Archean bimodal trondhjemite-basalt suits, Geology, 4, 596-600.

Barker, F., A. G. Arth, Z. E. Peterman, and I. Friedman (1976), The 1.7 and 1.8 b.y. old trondhjemites of the southern Colorado and Northern New Mexico, Geol. Soc. Am. Bull., 87, 632-643.

Barker, F., A. G. Arth, and T. W. Stern (1986), Evolution of the Coastal batholith along the Skagway traverse, Alaska and British Columbia. Am. Mineral, 71, 632-643.

Batanova, V. G., and O. V. Astrakhantsev (1992), Tectonic setting and genesis of the zoned mafic-ultramafic plutons of the northern Olyutorsky zone (Koryak Highlands), Geotektonika (in Russian), 2, 87-103.

Batanova, V. G., and O. V. Astrakhantsev (1994), Island-arc mafic-ultramafic plutonic complex of North Kamchatka, Proc. 29th Int't. Geol. Congr., D, pp. 129-143.

Bogdanov, N. A. (1988), Tectonics of the Deep Basins of Marginal Seas, Nedra, Moscow.

Bogdanov, N. A., and S. M. Tilman (1992), Tectonic map of northeastern Asia (scale 1:5000000), Committee on geodesy and cartography of the Ecology and Mineral resource Ministry of the Russian Federation, Moscow.

Bogdanov, N. A., and S. M. Tilman (1993), Tectonics and Geodynamics of Northeastern Asia (Explanatory notes to the Tectonic Map of Northeastern Asia, (scale 1:5000000), Institute of the Lithosphere RAS, Circum-Pacific Council for Energy and Mineral resources, Moscow.

Bogdanov, N. A., and V. E. Khain (2000a), Explanatory Notes to the Tectonic Map of the Okhotsk Sea Region (1: 2500000), Institute of the Lithosphere of Marginal Seas of RAS, Moscow.

Bogdanov, N. A., and V. E. Khain (2000b), Tectonic Map of the Okhotsk Sea Region (1:2500000), Institute of the Lithosphere of Marginal Seas of RAS, Moscow.

Bogdanov, N. A., V. S. Vishnevskaya, P. K. Kepezhinskas, N. A. Sukhov, and A. V. Fedorchuk (1987), Geology of Southern Koryak Highland (in Russian), Nauka, Moscow.

Brandon, M. T. (1992), Decomposition of fission-track grain age distribution, Am. J. Sci., 292, 535-564.

Brandon, M. T. (1996), Probability density plot for fission-track grain-age samples, Radiation Measurements, 26, 663-676.

Brandon, M. T., and J. A. Vance (1992), Fission-track ages of detrital zircon grains: implications for the tectonic evolution and Cenezoic Olympic subduction complex, Am. J. Sci., 292, 565-636.

Castro, A., I. Moreno-Ventas, J. D. de la Rosa (1991), H-type hybrid granitoids: a proposed version of the granite-type classification and nomenclature, Earth Sci. Review, 31, 237-253.

Chamov, N. P. (1996), Chemical composition of CretaceousPaleogene volcanogenic-sedimentary rocks of the GovenaKaragin block, southern Koryak upland, Lithology and Mineral Resources, 31, 348-359.

Chappell, B. W., and A. J. R. White (1974), Two contrasting granite types, Pacif. Geol., 8, 173-174.

Chekhovich, V. D., N. A. Bogdanov, I. R. Kravchenko-Berezhnoy, G. Yu. Averina, and A. Yu. Gladenkov (1990), Geology of the Western Bering Sea Region (in Russian), Nauka, Moscow.

Coleman, R. G., and Z. E. Peterman (1975), Oceanic plagiogranites, J. Geophys. Res., 80, 1099-1108.

Condie, K. C. (1993), Chemical composition and evolution of the upper continental crust: constraining results from surface samples and shales, Chem. Geol., 103, 1-37.

Crawford, M. B., and B. F. Windley (1990), Leucogranites of the Hymalaya/Karakorum: implication for magmatic evolution of within collisional belts and the study of collision-related leucogranite petrogenesis, J. Volcanol. Geothem. Res., 44, 119.

Dolmatov, B. K., S. A. Mel'nikova, and Yu. M. Stefanov (1969), Mezosoic deposits of Karaginski island (eastern Kamchatka), Doclady Earth Sciences (in Russian), 187, 1117-1119.

Dymond, J. (1981), Geochemistry of Nazca plate surface sediments: An evaluation of hydrothermal, biogenic, detrital, and hydrogenous sources, Geol. Society of America, 154, 133-173.

England, P. C., and B. Thompson (1984), Pressure - temperature
- time paths of regional metamorphism, J. Petrol, 25(4), 894955.

Ewart, A., and C. J. Hawkesworth (1987), The Pleistocene - recent Tonga - Kermadek arc lavas: interpretation of new isotopic and rare earth element data in terms of depleted mantle source, J. Petrol., 28, 43-57.

Falloon, T. J., D. H. Green, H. St. C. O'Neill, and W. O. Hibberson (1997), Experimental test of low degree peridotite partial melting compositions: implication for the nature of anhydrous near-solidus peridotite melts at $1 \mathrm{Gpa}$, Earth Planet. Sci. Lett., 152, 149-162.

Filatova, N. I. (1988), Periocenic volcanic belts (in Russian), Nedra, Moscow.

Floyd, P. A., and B. E. Leveridge (1987), Tectonic environment of the Devonian mode and geochemical evidence from turbiditic sandstones, J. Geol. Soc. London, 144, 531-542.

Garver, J. I., and M. T. Brandon (1994), Erosional denudation of the British Columbia Coast Ranges as determined from fissiontrack ages of detrital zircon from the Tofino basin, Olympic Peninsula, Washington, Geol. Soc. Amer. Bull., 106, 13981412.

Garver, J. I., and T. J. Scott (1995), Trace elements in shale as indicators of crustal provenance and terrane accretion in the southern Canadian Cordillera, GSA Bulletin, 107, 440-453.

Garver, J. I., P. R. Royce, and T. A. Smick (1996), Chromium and nickel of the Taconic foreland: a case study for the provenance of the fine-grained sediments with an ultramafic source, J. of Sedimentary Research, 66, 100-106.

Garver, J. I., A. V. Soloviev, P. J. J. Kamp, and M. T. Brandon (1999), Detrital zircon fission-track thermochronology: practical considerations and examples, in Fission-track analysis: theory and application, edited by S. Martin and R. Polino, Memorie di Scienze Geologiche, 51/2, 454-456, PAVODA.

Garver, J. I., A. V. Soloviev, M. E. Bullen, and M. T. Brandon (2000), Towards a more complete record of magmatism and exhumation in continental arcs, using detrital fission-track thermochronology, Phys. Chem. Earth (A), 25, 565-570.

Garver, J. I., A. V. Soloviev, M. T. Brandon, M. N. Shapiro, and J. K. Hourigan (2001), Tectonic and magmatic history of Kamchatka and NE Asia revealed through detrital zircon thermochronology of Cretaceous to Miocene deep-water strata, Kamchatka (Russia), VII Zonenshain International Conference on Plate Tectonics, p. 503, Nauchniy Mir, Moscow.

Gleadow, A. J. W., A. J. Hurford, and R. D. Quaife (1976), Fission-track dating of ziron: improved etching techniques, Earth Planet. Sci. Lett., 33, 273-276.

Gorton, M. P., and E. S. Schandl (2000), From continents to island arcs: a geochemical index of tectonic setting for arcrelated and within-plate felsic to intermediate volcanic rocks, The Canadian Mineralogist, 38, 1065-1073.

Harris, N. B. W., J. A. Pearce, and A. G. Tindle (1986), Geochemical characteristics of collision zone magmatism, in Collision Tectonics, Geological Society Special Publications, 19, edited by M. P. Coward and A. C. Ries, pp. 67-81.

Hochstaedter, A. G., P. K. Kepezhinskas, M. S. Defant, M. S. Drummond, and H. Bellon (1994), On the tectonic significance of arc volcanism in northern Kamchatka, J. Geol., 102, 639-654.

Hofmann, A. W. (1988), Chemical differentiation of the Earth: the relationship between mantle, continental crust, and oceanic crust, Earth Planet. Sci. Lett., 90, 297-314.

Kepezhinskas, P. K., and A. T. Savichev (1991), Geochemical stratification and evolution of early island-arc magma chambers, Geology of the Pacific ocean (in Russian), 1, 12-26.

Kepezhinskas, P. K., L. B. Efremova, and N. A. Sorokina (1991), Rare-earth distribution in plutonic complexes of early-stage island arc, Geochemistry International (in Russian), 4, 562-570. Kepezhinskas, P. K., R. N. Taylor, and H. Tanaka (1993), Geochemistry of plutonic spinels from the North Kamchatka arc: comparisons with spinels from other tectonic settings, Mineralogical Magazine, 57, 575-589.

Kravchenko-Berezhnoy, I. R., and Yu. V. Nazimova (1991), The Cretaceous ophiolites of Karaginski Island (the Western Bering 
Sea), Ofioliti, 16, 79-110.

Ledneva, G. V., A. V. Soloviev, and J. I. Garver (2000), Massifs of the heterogeneous mafic-ultramafic complex of the Olyutorsky zone, Koryak Mountains: Petrology and geodynamic aspects, Petrology, 8, 428-454.

Lin, P. N., R. J. Stern, and S. H. Bloomer (1989), Shoshonitic volcanism in northern Mariana arc 2, Large-ion-lithophile and rare earth element abundances: evidence for the source incompatible element enrichment in intraoceanic arcs, J. Geophys. Res., 94, 4497-4514.

Martin, H. (1986), Effect of steeper Archean geothermal gradient on geochemistry of subduction zone magmas, Geology, 14, 753756.

Martin, H. (1993), The mechanisms of petrogenesis of the Archean continental crust - comparison with modern processes, Lithos, 30, 373-388.

McLennan, S. M., S. Hemming, D. K. McDaniel, and G. N. Hanson (1993), Geochemical approaches to sedimentation, provenance, and tectonics, in Processes controlling the composition of clastic sediments, Geol. Soc. Amer. Spec. Paper, 284, edited by M. J. Johnsson and A. Basu, pp. 21-40. Naeser, C. W. (1979), Fission-track dating and geologic annealing of fission-tracks, in Lectures in Isotope Geology, edited by E. Jager and J. C. Hunziker, pp. 154-169, Springer-Verlag, Heidelberg.

Naeser, C. W., P. K. Zeitler, C. W. Naeser, and P. F. Cerveny (1987), Provenance studies by fission-track datind-etching and counting procedures, Nucl. Tracks Radiat. Meas., 13, 121-126.

O'Conner, J. I. (1965), A classification of quartz-rich igneous rocks based on feldspar ratios, U.S. Geol. Sur. Prof. Pap, $525 B$.

Pearce, J. A., N. B. W. Harris, and A. G. Tindle (1984), Trace element discrimination diagrams for the tectonic interpretation of granitic rocks, J. Petrol., 25, 956-983.

Peccerillo, A., and S. R. Taylor (1976), Geochemistry of Eocene calc-alkaline volcanic rocks from the Kastamonu area, northern Turkey, Contrib. Miner. Petrol., 58, 63-81.

Puzankov, Yu. M., O. N. Volynets, V. A. Seliverstov, G. N. Anoshin, M. G. Patoka, M. G. Valov, V. S. Uspenskiy, and M. N. Shapiro (1990), Catalog of geochemical data (igneous and metamorphic rocks of Kamchatka and Komandorsky Islands), in Transactions of the Institute of Geology and Geophysics of USSR Academy of Sciences, Geochemical Typification of Igneous and Metamorphic Rocks of Kamchatka (in Russian), vol. 390, edited by A. P. Krivenko, pp. 206-256, Siberian Branch of the USSR Academy of Sciences, Institute of Geology and Geophysics, Novosibirsk.

Robinson, A. C. J., B. J. Wood, and J. D. Blundy (1998), The beginning of melting of fertile and depleted peridotite at $1.5 \mathrm{Gpa}$, Earth Planet. Sci. Lett., 155, 97-111.

Rozen, O. M. (2001), Derived Granitoids and Their lower crustal residue: Numerical simulation of the collision-related Pliocene Tyrnyauz Granites, Greater Caucasus, Geochemistry International, 39, 487-505.

Rozen, O. M., D. Z. Zhuravlev, and S. M. Lyapunov (1994), Geochemical studies of sedimentary deposits of the Timan-Pechora province, Razvedka i Okhrana nedr (in Russian), (1), 18-21.

Rubenstone, J. L. (1984), Geology and geochemistry of early Tertiary submarine volcanic rocks of the Aleutian Islands and their bearing on the development of the Aleutian Island Arc, Ph.D. Thesis, Ithaka, NY, USA.

Seliverstov, N. I. (1998), Structure of the Floor of KamchatkaRelated Waters and Geodynamic of Articulation Zone of the Kuril-Kamchatka and Aleutian Island Arcs (in Russian), Nauchniy Mir, Moscow.

Shantser, A. E., and P. I. Fedorov (1997), Early Paleogene volcanism, in Transactions of the Geological Institute of the Russian Academy of Sciences, The Lower Paleogene of Western Kamchatka (Stratigraphy, Paleontology, Geological Events) (in Russian), vol. 148, edited by Yu. G. Leonov et al., pp. 117-128, GEOS, Moscow.

Shapiro, M. N. (1984), Rhyolitic and granitic clasts in the Cretaceous - Paleogene sequences of Karaginski Island: their com- position and source problem, Bulletin of Moscow Society of Naturalists, Geology series (in Russian), 59(5), 23-33.

Shapiro, M. N., and N. M. Petrina (1985), The new data on age of Karaginski Island (the Bering Sea), Geology of the Pacific ocean (in Russian), (1), 108-110.

Shapiro, M. N., A. V. Lander, and N. P. Chamov (2000), Provenance of sialic clastic material in the Eocene-Oligocene sequences of northeastern Kamchatka: implication for geodynamics of the Komandorsky basin, the Bering Sea, Geology of the Pacific ocean (in Russian), (19), 66-82.

Shapiro, M. N., A. V. Soloviev, J. I. Garver, E. A. Stcherbinina, G. V. Ledneva, and M. T. Brandon (2004), The age of terrigenous deposits of northeastern Karaginski Island (eastern Kamchatka), Stratigraphy and geological correlation (in Russian), 12(2), 90-101.

Shipilov, E. V. (1988), Structure of the sedimentary cover in the western Bering Sea, Izv. AN SSSR. Ser. Geology (in Russian), $1,120-128$.

Soloviev, A. V., M. T. Brandon, J. I. Garver, N. A. Bogdanov, M. N. Shapiro, and G. V. Ledneva (1998), Collision of the Olyutor Island Arc with the Eurasian Continental Margin: Kinematic and Age Aspects, Doklady Earth Sciences, 361, 632-634.

Soloviev, A. V., M. N. Shapiro, J. I. Garver, E. A. Shcherbinina, and I. R. Kravchenko-Berezhnoy (2002), New age data from the Lesnaya Group: A key to understanding the timing of arccontinent collision, Kamchatka, Russia, The Island Arc, 11, 79-90.

Stcherbinina, A. E. (1997), Nannoplankton from Paleogene Deposits in Eastern Kamchatka, Stratigraphy and Geological correlation, 5, 156-166.

Stolper, E. (1980), A phase-diagram for mid-ocean ridge basalts: preliminary results and implications for petrogenesis, J. Petrol., 74, 13-27.

Sukhov, A. N. (1983), Volcanic complex of the Olyutorsky Ridge, Izv. AN SSSR. Ser. Geology (in Russian), 10, 12-27.

Taylor, S. R., and S. M. McLennan (1985), The Continental Crust: its Composition and Evolution (An examination of geochemical record preserved in sedimentary rocks), Blackwell Scientific Publications. Oxford, London, Edinburgh, Boston, Palo Alto, Melbourne.

Wagner, G. A., and P. Van den Haute (1992), Fission-Track Dating, 285 pp., Kluwer Academic Publishers, Dordrecht.

White, A. J. R., and B. W. Chappel (1977), Ultrametamorphism and granitoid genesis, Tectonophysics, 43, 7-22.

Wyllie, P. J., W.-L. Huang, C. R. Stern, and S. Maaloe (1976), Granitic magmas: possible and impossible sources, water contents, and crystallization sequences, Can. J. Earth Sci., 13, 1007-1019.

Yagodzinski, G. M., R. W. Kay, O. N. Volynets, A. V. Koloskov, and S. M. Kay (1995), Magnesian andesites in the western Aleutian Komandorsky region: Implications for slab melting and processes in the mantle wedge, Geol. Soc. Am. Bull., 107, 505-519.

Yudovich, Ya. E. (1981), Regional geochemistry of sedimentary deposits (in Russian), Nauka, Leningrad.

G. V. Ledneva, Geological Institute, Pyzhevsky per. 7, Moscow 119035, Russia, e-mail: Ledneva@ilran.ru

J. I. Garver, J. Lederer, and K. T. Hollocher, Geology Department, Union College, Schenectady, NY 12308-2311, USA, e-mail: garverj@union.edu

M. N. Shapiro, Institute of the Earth Physics after A.A. Gamburtsev, RAS, B. Gruzinskaya ul. 10, Moscow 123810, Russia, e-mail: mns@uipe-ras.scgis.ru

M. T. Brandon, Dept. of Geology and Geophysics, Yale University, P.O. Box 208109, 210 Whitney Avenue, New Haven, CT 06520-8109; e-mail: mark.brandon@yale.edu

(Received 3 April 2004) 